Historic, Archive Document

Do not assume content reflects current scientific knowledge, policies, or practices. 

WTALIER-IRANIELIN

1980 


\section{CONTRACT \\ AND \\ GENERAL TRADE LIST \\ $\mathrm{OF}$ \\ “MERIT" FLOWER SEEDS}

\section{CROP of 1930}

To the Seed Trade only

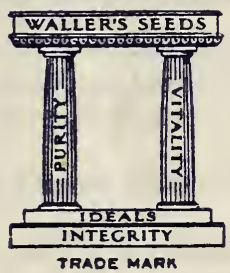

Waller-Franklin Seed Co.

WHOLESALE GROWERS

GUADALUPE, CALIFORNIA, U. S. A.

Cable Address "Waller, Guadalupe"

Code A. B. C. Fifth Edition Improved 


\section{Foreword}

TO THE TRADE:

We take pleasure in presenting our 1930 contract and general trade list.

An institution grows as it is capable of serving well its many customers: WE serve the discriminating trade what they demand: MERIT FLOWER SEEDS.

We are justly proud of the esteem and favor our stocks have attained, and devote all our energies to the perfecting of strains and the breeding up of new varieties.

We extend to the seed trade a most cordial invitation to visit our experimental and trial grounds and our extensive ranches, and personally acquaint themselves with the purity of our cultures.

We again welcome the opportunity to thank our patrons for their generous support in the past and will do our utmost to justify their continuance.

\section{GENERAL CONDITIONS OF DELIVERY}

For every order, we agree to plant sufficient land to produce the desired quantity of seed under normal conditions.

Should the outcome of the crop be lower than our estimation, orders will be filled pro rata only.

Crop failure or any other cause beyond our control releases us entirely from delivery.

We give no warranty, express or implied, as to description, purity, productiveness, or any other matter of any seeds we sell, and we will not be in any way responsible for them.

\section{TERMS}

All seeds are quoted f. o. b. Guadalupe, California, for delivery after harvest crop of 1930, 60 days acceptance net, less $2 \%$ cash thirty days.

All overdue accounts subject to sight draft without further notice.

Bags and containers will be charged at cost and are not returnable.

While we are confident prices given in this catalog are very reasonable, we shall be pleased to have the opportunity of meeting any reasonable competition of houses of repute.

No order filled for less than a quarter pound except where ounce prices are quoted.

We reserve the right to change any prices in this list after May 1st, 1930.

WALLER-FRANKLIN SEED COMPANY 


\section{WALLER-FRANKLIN FORMULA MIXTURES}

As the result of years of experimenting, our research department has perfected certain color blends of various mixtures.

These mixtures were subject to the criticism of visiting seedsmen for several years and when unanimously approved were considered MASTER FORMULAE.

Mixtures grown from these Master Formulae are listed in this catalog as "WALLER-FRANKLIN FORMULA MIXTURES."

\section{PHOTOGRAPHS}

We have a large collection of photographs of annuals and perennials suitable for catalog work and we will be pleased to send selections for your inspection. Price $\$ 2.00$ per print. 


\title{
NOVELTIES AND RECENT INTRODUCTIONS
}

\author{
By \\ WALLER-FRANKLIN
}

We devote a great deal of time and attention to the perfecting of original strains and also to the development of new colors and types of flowers and each year have had the pleasure of introducing a number of outstanding novelties.

We devote fifty acres of our very finest land to Experimental Grounds and we have many worth while novelties in various stages of development. An announcement regarding our novelties for 1930 will be made in August.

\section{Novelties Introduced By Us in 1929}

$* * * * * * * *$

\section{THREE NEW EARLY FLOWERING SWEET PEAS}

VALENCIA-Color, bright orange, sunproof, of a slightly softer tone than Orange King but having what all other orange Sweet Peas lackImmense Vigor in growth, length of stem and size of blooms. Where other orange Sweet Peas throw only two and three blooms to a spray, VALENCIA throws three and four. Price per oz. $\$ 1.00$, per $1 / 4$ lb. $\$ 3.00$, per lb. $\$ 10.00$.

MAJESTIC ROSE-Color, deep brilliant rose pink of the popular color of Zvolanek's Rose, but of a richer, deeper tone. The color in MAJESTIC ROSE is more evenly diffused than in any other Sweet Pea in this color class and lacks the white at the base of the standard and wings which others of this color have. This improvement in color gives it a brilliance which is quite new. In vigor of growth, length of stem, size of bloom, MAJESTIC ROSE excels all other early Sweet Peas. Price per oz. $\$ 1.50$, per $1 / 4 \mathrm{lb} . \$ 4.50$, per $\mathrm{lb} . \$ 15.00$.

BLUE BONNET-Color, a fine deep blue. The color is evenly distributed, with not the slightest trace of mauve. By careful comparison the Color and Vigor of BLUE BONNET are far superior to any other blue on the market. It has very long stems with four blossoms to a stem. Price per oz. $\$ 1.00$, per $1 / 4 \mathrm{lb}$. $\$ 3.00$, per $\mathrm{lb}$. $\$ 10.00$. 


\title{
NOVELTIES AND RECENT INTRODUCTIONS
}

\author{
By \\ WALLER-FRANKLIN
}

$* * * * * * * *$

Novelties Introduced By Us in 1929

COSMOS EARLY EXPRESS PINK-An extra early Cosmos flowering in from forty-five to fifty days from the time seed is planted. The plant grows about two and one-half to three feet high and is covered with medium sized bright pink flowers. Price per oz. $\$ 0.50$, per $1 / 4$ lb. $\$ 1.50$, per lb. $\$ 5.00$.

LARKSPUR EMPEROR BRILLIANT CARMINE-A delightful addition to this group, which grows only about twenty-four inches high. The color is identical to Lustrous Carmine in the Tall Branching group and makes a good companion to Pink Delight now so popular. Price per oz. $\$ 0.50$, per $1 / 4$ lb. $\$ 1.50$, per lb. $\$ 5.00$.

LARKSPUR WHITE SPIRE-A new dazzling pure white double Larkspur with the improved upright delphinium-like habit of our last year's novelty-Exquisite Pink Improved. This is a relcome addition to the new group of column-like Larkspurs, as the long straight upright stems make it superior to all other white Larkspurs as a cut flower. Price per oz. $\$ 1.00$, per $1 / 4 \mathrm{lb}$. $\$ 3.00$, per lb. $\$ 10.00$.

STOCK EARLY GIANT IMPERIAL OLD ROSE-A very beautiful shade of old rose similar to the very popular Old Rose in the Nice groupthis color has been lacking in the Giant Imperial group. It is very double and has very large individual florets. Price per oz. $\$ 3.50$, per $1 / 4$ lb. $\$ 10.50$, per lb. $\$ 35.00$.

VERBENA DWARF COMPACT FIREBALL-A dwarf compact Verbena about six inches high literally covered with bright scarlet blooms, excellent for borders, beds, or pot plants and comes absolutely true from seed. Price per oz. $\$ 2.00$, per $1 / 4 \mathrm{lb}$. $\$ 6.00$, per lb. $\$ 20.00$. 


\title{
NOVELTIES AND RECENT INTRODUCTIONS
}

\author{
By \\ WALLER-FRANKLIN \\ ********** \\ Novelties Introduced By Us in 1928 \\ **********
}

\section{LARISSPUR EXQUISITE PINK IMPROVED}

Award of Merit Royal Horticultural Society, Wisley Trials, 1929

Exquisite in color. Perfection in form. Purity of strain.

Eight years ago we introduced Larkspur Exquisite Pink; it at once became the standard of excellence in Larkspurs in spite of the fact that the strain was not true.

After a further eight years of effort we have perfected a new strain, true Exquisite Pink in color with a wonderful improved new form. An upright habit similar to our perennial Delphinium and best of all, this new improved strain comes practically $100 \%$ true. The color is a beautiful soft pink shaded salmon. Price per oz. $\$ 0.80$, per $1 / 4 \mathrm{lb}$. $\$ 2.50$, per Ib. $\$ 8.00$.

\section{LARKSPUR EXQUISITE ROSE}

\section{Award of Merit Royal Horticultural Society, Wisley Trials, 1929}

A beautiful shade of rose pink, several tones deeper than Exquisite Pink Improved but with the same new improved Delphinium form and coming quite true. Price per oz. $\$ 0.80$, per $1 / 4 \mathrm{lb}$. $\$ 2.50$, per lb. $\$ 8.00$.

\section{NEW EARLY FLOWERING SPENCER SWEET PEA}

PINK CHARM-A light rose pink, practically self-colored with wings and standard somewhat lighter toward the outer edges, giving the whole a most charming effect. A vigorous grower, with a long blooming period and when other varieties are through blooming, Pink Charm is still good. A splendid variety for florists. Price per oz. $\$ 0.50$, per $1 / 4$ lb. $\$ 1.50$, per lb. $\$ 5.00$. 


\title{
NOVELTIES AND RECENT INTRODUCTIONS
}

\author{
By \\ WALLER-FRANKLIN \\ $* * * * * * * *$ \\ Novelties Introduced By Us in 1928.
}

HELICHRYSUM MONSTROSUM FL. PL. SILVERY PINK-A soft pleasing shade of delicate silvery cameo pink. The dainty delicate color is something quite new and heretofore unknown in Helichrysums. The blooms are quite large, slightly over two inches in diameter. This will prove to be a very good commercial variety. Price per $1 / 4 \mathrm{lb}$. $\$ 0.90$, per lb. $\$ 3.00$.

STOCK EARLY GIANT IMPERIAL ELK'S PRIDE-Elk's Pride is a new shade in the Early Giant Imperial group, being an intense royal purple of vigorous growth, attaining a height of twenty-four to thirty inches. It is very early blooming, the florets are quite large and sweetly scented. The strain is very double. Price per oz. \$3.50, per $1 / 4 \mathrm{lb}$. $\$ 10.50$, per lb. $\$ 35.00$.

VERBENA HYBRIDA GRANDIFLORA ROYALE-This is a Verbena of enormous size, the individual florets measuring over an inch and a quarter in diameter. The color of this new Verbena is a rich deep royal blue with a large creamy yellow eye. A wonderful acquisition to our group of Grandiflora Verbenas. Price per oz. $\$ 1.00$, per 1/t lb. $\$ 3.00$, per lb. $\$ 10.00$.

DELPHINIUM WALLER-FRANKLIN HOLLYHOCK STRAIN (WreXham Type)-These are the greatest advance in Delphiniums. Their graceful spire shaped spikes of enormous size and beauty of form are rapidly bringing them to the front as the most popular flower for cutting or the garden. This mixture contains all the colors in Delphiniums, including both double and single flowers and has been greatly praised by professional Delphinium growers. Price per oz. $\$ 4.00$, per $1 / 4 \mathrm{lb}$. $\$ 12.00$, per lb. $\$ 40.00$. 


\title{
NOVELTIES AND RECENT INTRODUCTIONS
}

\author{
By \\ WALLER-FRANKLIN
}

NEW EARLY FLOWERING SPENCER SWEET PEA

IMPERIAL PINK-Imperial Pink is the last word in Early pink Sweet

Peas and this new variety, we prophesy, will be the favorite pink among florists and in our gardens for many years to come. A clear pink of enormous size bred up from a line of strong and tested parentage, insuring a vigorous growth, long stems and an abundance of blooms over a long period. Price per $1 / 4 \mathrm{lb}$. $\$ 0.90$, per $\mathrm{lb}$. $\$ 3.00$.

GAILLARDIA GRANDIFLORA, PORTOLA HYBRIDS-This superb new strain of Gaillardia bears flowers of very large size, the color ranging through shades of bronzy red with the characteristic golden tipped petalage and robust vigor of the well known Portola variety. A splendid cut flower. Price per oz. $\$ 1.00$, per $1 / 4 \mathrm{lb} . \$ 3.00$, per lb. $\$ 10.00$.

HELICHRYSUM MONSTROSUM FL. PL. ROSE QUEEN-Immense flowers of a rich deep rose. Extremely beautiful under artificial light. A wonderful addition to the Helichrysum group. Price per $1 / 4 \mathrm{lb}$. $\$ 0.90$, per lb. $\$ 3.00$.

SCABIOSA GRANDIFLORA FL. PL. PEACH BLOSSOM-"Highly Commended" by the Royal Horticultural Society of Great Britain. A beautiful shade of Peach Blossom pink, large size and long stems. An excellent cut flower. Price per $1 / 4 \mathrm{lb}$. $\$ 0.90$, per lb. $\$ 3.00$.

SCABIOSA GRANDIFLORA FL. PL. SHASTA-A pure white of enormous size, the flowers measuring three and a half inches across with extremely long stems. Price per $1 / 4 \mathrm{lb}$. $\$ 0.90$, per $\mathrm{lb}$. $\$ 3.00$.

STOCK EARLY GIANT IMPERIAL ANTIQUE COPPER-Rich Hellebore red, overlaid with copper, the whole giving a very pleasing reddish antique copper effect. The strain is very double with individual florets two inches in diameter on long stems. Price per oz. $\$ 3.50$, per $1 / 4 \mathrm{lb}$. $\$ 10.50$, per lb. $\$ 35.00$.

STOCK EARLY GIANT IMPERIAL GOLDEN ROSE-A rich light rose enhanced by a pleasing golden effect in the center of each individual floret. Very double, large flowers on long stems. A wonderful cut flower. Price per oz. $\$ 3.50$, per $1 / 4 \mathrm{lb}$. $\$ 10.50$, per lb. $\$ 35.00$.

VERBENA HYBRIDA GRANDIFLORA ETNA-Large trusses of intense Paul Crampbell geranium red with a small creamy yellow eye. The individual florets are over one inch in diameter. Price per oz. $\$ 1.00$, per $1 / 4 \mathrm{lb}$. $\$ 3.00$, per lb. $\$ 10.00$. 


\section{SPECIALTIES AND FLORISTS' STRAINS}

ALYSSUM PROCUMBENS-Carpet of Snow. A very select strain grown by us with the greatest of care, habit two and a half inches high and about twelve to eighteen inches in diameter, and has the appearance of a nice flat pancake. The blooms are massed, completely hiding all foliage, and a field of this in bloom has the appearance of being covered with snow. Price per $1 / 4 \mathrm{lb}$. $\$ 0.75$, per lb. $\$ 2.50$.

CALENDULA, F1. P1. BALL'S GOLD IMPROVED-Dark golden yellow with long stems. A companion to Calendula The Ball. Price per $1 / 4$ lb. $\$ 1.50$, per lb. $\$ 0.00$.

CALENDULA, F1. P1. ORANGE KING-Double, dark orange red, dark center; a very select strain grown by us with the greatest of care for our customers in the seed trade who make a specialty of catering to the florist trade. The plants in the open ground under ordinary field cultivation attain a growth of eighteen inches high and produce blossoms three and a half inches in diameter. Price per $1 / 4 \mathrm{lb} . \$ 0.30$, per lb. $\$ 1.00$.

CALENDULA, F1. P1. THE BALL-A special florists' strain, color orange with a light center; long stems with very large uniformlydouble flowers. This is an exceptionally fine Calendula for forcing. Price per $1 / 4$ lb. $\$ 1.50$, per lb. $\$ 5.00$.

\section{CANDYTUFT, GIANT HYACINTH FLOWERED WHITE EXTRA}

SELECT-This is a very superior strain, it grows with us about $1 \frac{1}{2}$ feet high, with enormous trusses of blooms. It has been one of our specialties for a number of years and every effort is made to keep it at a very high state of perfection. We believe it is superior to any other strain on the market. Price per $1 / 4 \mathrm{lb}$. $\$ 0.90$, per $\mathrm{lb} . \$ 3.00$. 


\section{SPECIALTIES AND FLORISTS' STRAINS}

\section{CARNATION CHABAUD'S ENFANT DE NICE}

Carnation Enfant de Nice is the result of a cross between Carnation Chabaud's Giant Improved and Carnation Perpetual. The plants are of robust, upright habit, with long, strong stalks bearing flowers $2 \frac{1}{2}$ to 3 inches in diameter. This strain is magnificent and is so far, the last word in annual Carnations. The color comes about $75 \%$ true from seed and about $90 \%$ of the plants come double.

\begin{tabular}{|c|c|c|c|}
\hline & $\begin{array}{c}\text { Price } \\
1 / 16 \mathrm{oz} .\end{array}$ & $\begin{array}{l}\text { Price } \\
1 / 4 \mathrm{oz} .\end{array}$ & $\begin{array}{l}\text { Price } \\
\text { Peroz. }\end{array}$ \\
\hline Red.. &..$\$ 1.00$ & $\$ 3.00$ & $\$ 10.00$ \\
\hline Rose...... & $\ldots 1.00$ & 3.00 & 10.00 \\
\hline Salmon. & $\ldots 1.00$ & 3.00 & 10.00 \\
\hline White.... & $\ldots 1.00$ & 3.00 & 10.00 \\
\hline Mixed. & $\ldots \quad .50$ & 1.50 & 5.00 \\
\hline
\end{tabular}

CENTAUREA CYANUS--Three fine varieties.

Price Price $1 / 4 \mathrm{lb}$. per lb.

BLUE Fl. Pl., most popular variety. $\$ .40$

$\$ 1.25$

ROSE Fl. Pl. (Waller-Franklin), exceptionally fine............... $\quad .40 \quad 1.25$

WHITE Fl. Pl., pure white and very double.

$.40 \quad 1.25$

CENTAUREA IMPERIALIS AMARANTH RED (Waller-Franklin) - A new color in this group giving a wonderful effect under artificial light. Price per $1 / 4 \mathrm{lb} . \$ 1.50$, per lb. $\$ 5.00$.

\section{COSMOS EXTRA EARLY DOUBLE CRESTED}

This beautiful group of Double Cosmos is variable as to percentage of doubleness, which does not often exceed fifty per cent. Both double and single flowers will appear on the same plant.

\begin{tabular}{|c|c|c|c|}
\hline & $\begin{array}{l}\text { Price } \\
\text { per oz. }\end{array}$ & $\begin{array}{l}\text { Price } \\
1 / 4 \mathrm{lb} \text {. }\end{array}$ & $\begin{array}{l}\text { Price } \\
\text { per lb. }\end{array}$ \\
\hline Carnelia (Waller-Franklin) crimson.. & $\$ 1.80$ & $\$ 5.40$ & $\$ 18.00$ \\
\hline a (Waller-Franklin) pink. & .1 .80 & 5.40 & 18.00 \\
\hline ller-Franklin) white.. & 1.80 & 5.40 & 18.00 \\
\hline noice & 1.20 & 3.60 & 12.0 \\
\hline
\end{tabular}




\section{SPECIALTIES AND FLORISTS' STRAINS}

DELPHINIUM BELLADONNA IMPROVED (Cliveden Beauty) light blue, larger flowered and a stronger grower than the original Belladonna. Price per oz. $\$ 3.00$, per $1 / 4$ lb. $\$ 9.00$, per lb. $\$ 30.00$.

DELPHINIUM BELLAMOSUM, Dark Blue-This Delphinium has the Belladonna habit and the Formosum dark blue color, hence the name Bellamosum. Price per $1 / 4 \mathrm{lb}$. $\$ 4.50$, per lb. $\$ 15.00$.

DELPHINIUM HYBRIDS XXX MIXED (Waller-Franklin)-For a number of years we have been carefully selecting Delphinium varieties with a view to making a mixture which would contain all the most beautiful shades of color obtainable in a perfect blend. Various formulae were tried out in our Experimental Grounds over a period of several years with the result that we now have a strain of Mixed Delphinium Hybrids on which we can put our highest mark of approval: "XXX." Price per oz. $\$ 1.50$, per $1 / 4 \mathrm{lb}$. $\$ 4.50$, per lb. $\$ 15.00$.

\section{DELPHINIUM}

Hybrids XXX Art Shades (Waller-Franklin)

" XXX Deep Blue Shades (Waller-Franklin)

“ XXX Light Blue Shades (Waller-Franklin)

" XXX Mid-Blue Shades (Waller-Franklin)

Price per oz. $\$ 2.00$, per $1 / 4 \mathrm{lb}$. $\$ 6.00$, per lb. $\$ 20.00$.

GYPSOPHILA ELEGANS GRANDIFLORA, PARIS MARKET-An improved strain of this popular florist flower, the plants grow about the same height as the older type, but the flowers are much larger and are pure white, without the objectionable veining of the older type. Price per $1 / 4 \mathrm{lb} . \$ 0.15$, per lb. $\$ 0.50$.

MARIGOLD TALL AFRICAN DOUBLE, ORANGE PRINCE (Fistulosa) -An especially fine tall branching strain, bearing enormous flowers of the quilled type. Price per $1 / 4 \mathrm{lb}$. $\$ 1.50$, per lb. $\$ 5.00$. 


\section{SPECIALTIES AND FLORISTS' STRAINS}

MIGNONETTE MACHET, NEW YORK MARKET-A magnificent strain of this very popular variety. Price per $1 / 4 \mathrm{lb}$. $\$ 1.50$, per $\mathrm{lb}$. $\$ 5.00$.

PETUNIA GIANTS OF CALIFORNIA, CHOICE MIXED-The largest and finest of all single Petunias. Price per $1 / 4 \mathrm{oz}$. $\$ 5.40$, per oz. $\$ 18.00$.

POPPY SHIRLEY AMERICAN LEGION (Waller-Franklin)-"Highly commended" by the Royal Horticultural Society of Great Britain. This new single Shirley is a dazzling orange scarlet of enormous size, borne on long stout stems. The plants are of very erect robust growth. Price per $1 / 4$ lb. $\$ 0.40$, per lb. $\$ 1.25$.

SCHIZANTHUS GRANDIFLORUS WALLER-FRANKLIN GIANT FLOWERING HYBRIDS-An extra select hothouse strain. Price per oz. $\$ 2.50$, per $1 / 4$ lb. $\$ 7.50$, per lb. $\$ 25.00$.

\section{STOCK EARLY GIANT IMPERIAL (Improved Bismarck)}

An especially meritorious class. Growing from twenty-four to thirty inches tall; of branching habit. Very early blooming and producing a high percentage of doubles.

Antique Copper (New) (Waller-Franklin) rich hel-

Price Price Price
per oz. $1 / 4 \mathrm{lb}$. per lb. lebore red

$\$ 3.50$

2.00

2.00

2.00

2.00

2.00

2.00

3.50

2.25

2.00

Flesh.

Golden Rose (New) (Waller-Franklin) light golden rose.

Lavender.

Lilac. 2.00

2.00

Mauve Lavender.

Old Rose (New) (Waller-Franklin) beautiful shade of old rose

Rose, deep rose pink

2.00

White.

2.00

1.80

Choice Mixed.

$\begin{array}{rr}\$ 10.50 & \$ 35.00 \\ 6.00 & 20.00 \\ 6.00 & 20.00 \\ 6.00 & 20.00 \\ 6.00 & 20.00 \\ 6.00 & 20.00 \\ 6.00 & 20.00 \\ 10.50 & 35.00 \\ 6.75 & 22.00 \\ 6.00 & 20.00\end{array}$

10.50

35.00

$6.00 \quad 20.00$
6.00

$6.00 \quad 20.00$

$6.00 \quad 20.00$

$10.50 \quad 35.00$

$6.00 \quad 20.00$

$6.00 \quad 20.00$

$5.40 \quad 18.00$ 


\section{SPECIALTIES AND FLORISTS' STRAINS}

STOCK PERPETUAL BRANCHING (Giant Perfection)

CANARY YELLOW (Creole). Award of Merit, R. H. S. ROSE, rose pink. Award of Merit, R. H. S.

SILVERY LILAC (Empress Augusta Victoria). Award of Merit, R. H. S.

These are three exceptionally fine Stocks which produce a very high percentage of double flowers. They each received an Award of Merit from the Royal Horticultural Society of Great Britain. Price per oz. $\$ 1.60$, per $1 / 4 \mathrm{lb}$. $\$ 4.80$, per lb. $\$ 16.00$.

\section{VERBENA HYBRIDA GRANDIFLORA}

A magnificent new strain of Verbenas, of robust compact habit and free blooming. The trusses are immense, the individual florets measuring an inch and over in diameter.

BLUE (Waller-Franklin) white eye.

COCCINEA (Waller-Franklin) scarlet, small eye.

ETNA (New) (Waller-Franklin) Paul Crampbell geranium red with creamy yellow eye.

GIANT SALMON PINK.

LUCIFER (Waller-Franklin) vivid scarlet self, nearly twice as large as the older type.

LUMINOSA (Waller-Franklin) luminous flame pink, shading to salmon.

ROSE CARDINAL (Waller-Franklin) carmine rose with large white eye.

ROSEA STELLATA (Waller-Franklin) a pure rose pink, with large white eye.

ROYALE (New) (Waller-Franklin) royal blue, creamy yellow eye.

VIOLACEA STELLATA, deep violet, white eye.

WHITE (Waller-Franklin) immense size.

Price per $1 / 4 \mathrm{lb}$. $\$ 3.00$, per lb. $\$ 10.00$.

ZINNIA ELEGANS CUT AND COME AGAIN-A group with double, perfectly formed, medium sized flowers and bred with long stems; exceptionally fine for cut flowers. This is a "Cut and Come Again" group and is especially fine for massing in the garden and produces a continuous succession of cut flowers throughout the Summer.

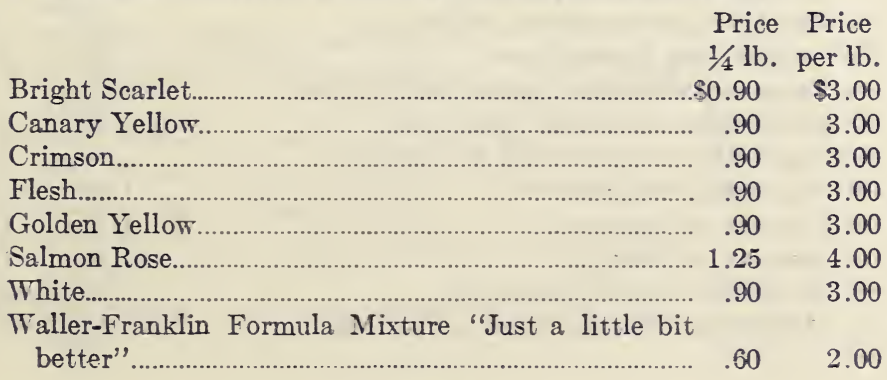


ABRONIA UMBELLATA, (Sand Verbena) pink, fragrant....\$0.60 $\$ 2.00$

AGATHEA COELESTIS, blue marguerite.

\section{ALYSSUM}

Maritimum (A. Benthami), sweet white......................... $\quad .30$

Compactum Erectum Little Gem.

Lilac Queen Improved, an extra select strain, true to color and form.

Lutescens, creamy yellow

Minimum.

Procumbens, Carpet of Snow, Waller-Franklin Special Strain.....

Saxatile Compactum, golden yellow.

\section{AMARANTHUS}

Caudatus (Love Lies Bleeding), red.............................. $\quad .10$

Tricolor (Joseph's Coat), yellow, green, red.................. $\quad .75$

\section{ANCHUSA}

Annual, Blue Bird (New) compact, indigo blue.............. 1.50

Capensis, dwarf forget-me-not-blue flowers.

Italica, Dropmore Variety, gentian blue (Reselected).. 1.00

\section{ANTIRRHINUM MAJUS GRANDIFLORUM (Snapdragon)}

Brilliant Rose, rose pink.

Cardinal, dazzling scarlet.

Coral Rose.

Crimson King, rich crimson..

Fire King, orange scarlet, white tube..

Golden King, giant yellow.

Harmony, terra cotta orange, shaded rose.

Pink Venus, flesh pink, white tube.

Queen Victoria Improved, pure white

Rose Queen, soft pink. 


\section{ANTIRRHINUM MAXIMUM}

This wonderful new group of Antirrhinums far surpasses anything yet introduced. In habit they are taller than the Majus group, growing about five feet high. The flowers are of magnificent size.

\section{Price}

per oz.

Apple Blossom, rosy pink, white tube.........\$1.20 $\$ 3.60 \quad \$ 12.00$

Canary Bird (Florists' Strain) canary yellow.... $1.50 \quad 4.50 \quad 15.00$

Copper King (Florists' Strain) bronzy copper... $1.50 \quad 4.50 \quad 15.00$

Old Gold (Florists' Strain).............................. $1.50 \quad 4.50 \quad 15.00$

Purple King, deep garnet................................... $1.00 \quad 3.00 \quad 10.00$

Snowflake, white..................................................... $1.20 \quad 3.60 \quad 12.00$

The Rose (Florists' Strain) begonia rose........... $1.50 \quad 4.50 \quad 15.00$

$\begin{array}{llll}\text { Maximum Mixed, all varieties............................ } & .75 & 2.25 & 7.50\end{array}$

\section{ANTIRRHINUM NANUM GRANDIFLORUM}

Amber Queen, amber, suffused chamois....................... $1.80 \quad 6.00$

Carmine Queen, brilliant rose carmine......................... $1.80 \quad 6.00$

Climax, orange shading to yellow in lip...................... 2.50 $\quad 8.00$

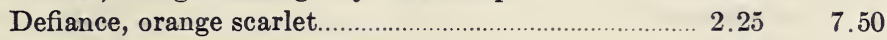

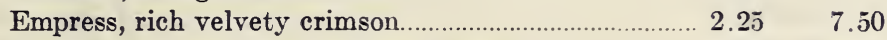

Fawn, terra cotta pink, suffused yellow....................... 1.25 $\cdots 4.00$

Firebrand, bright scarlet........................................... $1.50 \quad 5.00$

Gloria (Florists' Strain) rich deep rose pink .............. 2.25 $\quad 7.50$

Golden Queen, clear yellow.......................................... 1.25 4.00

Majestic Orange King, terra cotta................................. $6.00 \quad 20.00$

Majestic Twilight, rosy salmon....................................... $6.00 \quad 20.00$

Mauve Queen, lilac mauve.......................................... $1.50 \quad 5.00$

Nelrose (Florists' Strain) deep rose pink ..................... $1.50 \quad 5.00$

Peerless Pink (Waller-Franklin) (Florists' Strain) cameo pink ..................................................................... $3.00 \quad 10.00$

Philadelphia Pink (Florists' Strain) ............................ 2.25 $\quad 7.50$

Pink Perfection (Waller-Franklin) Hermosa pink, suffused

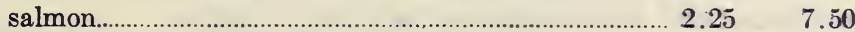

Prima Donna, terra cotta pink, white tube.................. $1.50 \quad 5.00$

Purity (Florists' Strain) pure white................................ 1.25 4.00

Rose Doré, rose, suffused orange................................. $1.50 \quad 5.00$

Silver Pink (Waller-Franklin) (Florists' Strain) pearly pink self........................................................................ $3.00 \quad 10.00$

Waller-Franklin Formula Mixture, "Just a little bit better". 
AQUILEGIA (Columbine)

Per oz.

Califomica Hybrida, golden yellow, red spur...\$1.00

$\$ 3.00 \$ 10.00$

Chrysantha, yellow.

1.00

$3.00 \quad 10.00$

Coerulea, Rocky Mountain Blue Columbine...... 2.00

$6.00 \quad 20.00$

Long Spurred Blue Shades.

3.50

$10.50 \quad 35.00$

西

Orange and Scarlet shades

3.50

10.50

35.00

" $"$

Pink Shades.

2.50

7.50

25.00

"

Rose Queen

2.50

7.50

25.00

Snow Queen, white.

3.50

$10.50 \quad 35.00$

"

Waller-Franklin Hybrids Mixed.. 2.50

$\begin{array}{ll}7.50 & 25.00\end{array}$

Hybrids Mixed

1.80

$5.40 \quad 18.00$

ARCTOTIS GRANDIS (African Lilac Daisy).

ARGEMONE (Mexican Prickly Poppy)

Platyceras Rosea.

Hybrida Grandiflora, Mixed

ARMERIA Formosa Hybrida (Giant Thrift) rose

\section{ASTER AMERICAN BEAUTY}

Azure Fairy.

Peach Blossom.

Purple

Rose.

Shell Pink (September Beauty).

White.

Choice Mixed

$2.10 \quad 7.00$

\section{ASTER CALIFORNIA GIANTS}

Dark Purple.

Deep Rose.

4.50

15.00

Light Blue.

4.50

15.00

Peach Blossom.

4.50

15.00

White.

Choice Mixed

ASTER GIANT BRANCHING COMET OR CREGO

Crimson.

Lavender.

Light Blue

Purple

Rose.

Shell Pink

White. 
ASTER QUEEN OF THE MARKET

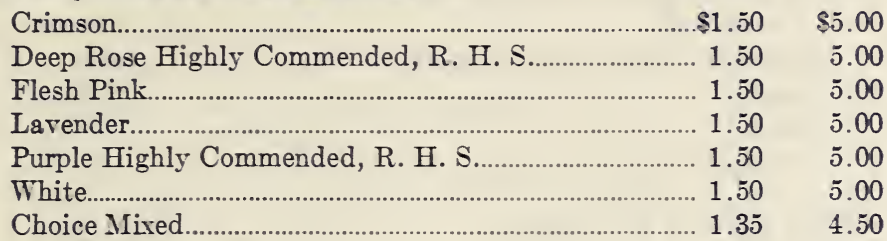

\section{ASTER EARLY ROYAL}

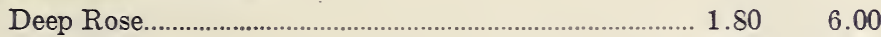

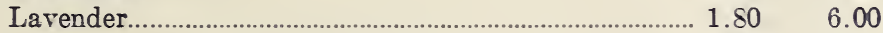

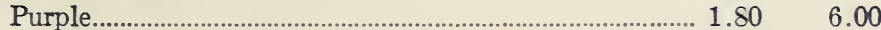

Shell Pink ............................................................................... $1.80 \quad 6.00$

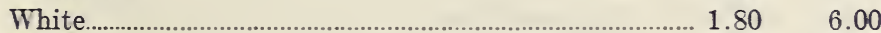

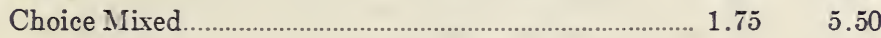

ASTER KING

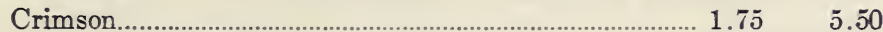

Lavender Highly Commended, R. H. S........................... 1.75 5.50

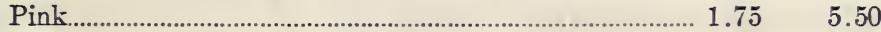

Rose................................................................................. 1.75

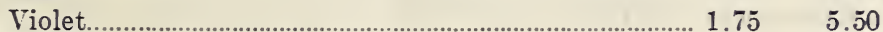

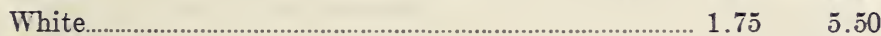

Choice Mixed ………………………………………..... $1.50 \quad 5.00$

\section{ASTER LATE BRANCHING}

Crimson.................................................................... $1.75 \quad 5.50$

Deep Rose Highly Commended, R. H. S...……………... 1.75 5.50

Lavender Highly Commended, R. H. S...…………….... 1.75 5.50

Purple ................................................................................... 1.75

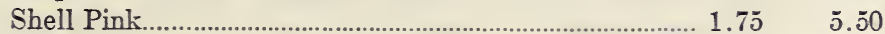

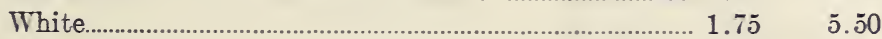

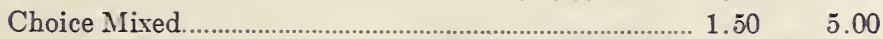

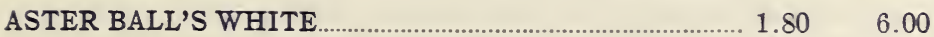

ASTER HEART OF FRANCE, most brilliant red Aster........ $3.60 \quad 12.00$

ASTER MORETON PINK ……………………………........... $1.80 \quad 6.00$

ASTER PEERLESS PINK Highly Commended, R.H.S. $1.65 \quad 5.50$

ASTER CHINENSIS TALL SINGLE GIANT FLOWERED

Apple Blossom, flesh pink ………………………………...... .50 $\quad .50$

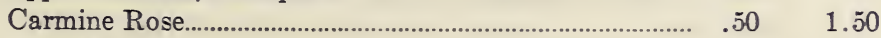

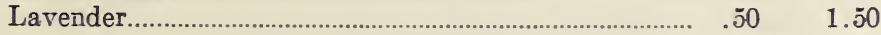


ASTER CHINENSIS TALL SINGLE GIANT FLOWERED -Continued.

Marechal Joffre, crimson scarlet.................................... $\$ 0.50 \quad \$ 1 . \tilde{50}$

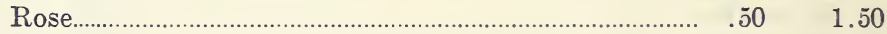

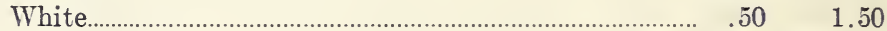

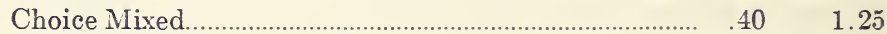

\section{ASTER SINGLE ELEGANCE}

Blue

Lavender........................................................................... $\quad .90$

Rose.

White

Choice Mixed.

BALLOON VINE (Cardiospermum) ....................................... . .25

BARTONIA AUREA, golden yellow.

BEANS SCARLET RUNNER, Scarlet Emperor.

BIDENS HUMULIS: See Cosmos Miniature Yellow.

BOLTONIA LATISQUAMA, delicate pink

\section{BROWALLIA ELATA}

Blue

White

Mixed

\section{CALENDULA: Flora Plena (Pot Marigold)}

Ball's Gold Improved (Florists' Strain) golden yellow.. 1.50

Ball's Orange Improved (Florists' Strain).

Ball's Supreme (Florists' Strain) cadmium orange, dark brown center.

Campfire (Sensation)

Favorita, cream striped yellow.

Golden King (Jaune Vif) cadmium orange, light center.

Lemon Queen (Sulphurea) sunflower yellow.................... . .20

Meteor, creamy white, striped orange............................... . .20

Nankeen (Le Proust) cream, flushed apricot.................. $\quad .20$

Orange King (Florists' Strain) dark orange red, dark

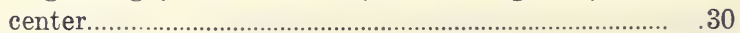

Trianon (Royal Marigold) yellow, striped orange, dark center. 


\section{CALLIOPSIS TALL : Bicolor}

Atrosanguinea, blood red.................................................. $\quad .40$

Marmorata, yellow and maroon marbled........................ $\quad .40$

1.25

Nigra Speciosa, dark brown.............................................. .40

Splendens, golden, brown center, extra large flowers...... . .40

Tinctoria, yellow, maroon center........................................... $\quad .30 \quad 1.00$

Hybrida Semi Plena Mixed................................................... $\quad .40 \quad 1.25$

Tall Annual Varieties Mixed.................................................... $\quad .25 \quad .90$

\section{CALLIOPSIS DWARF}

Bicolor Crimson King (Purpurea)..................................... $\quad .55 \quad 1.75$

" Dazzler, golden yellow, maroon center................ $\quad .55 \quad 1.75$

" Gold Ray, golden, maroon center.......................... $\quad .55 \quad 1.75$

“ Marmorata, yellow and maroon marbled........... $\quad .55 \quad 1.75$

“ Nana, yellow, brown center................................... $\quad .55$

“ Tiger Star (Radiata Tigrina) extra select........ $\quad .55 \quad 1.75$

“ Dwarf Annual Varieties Mixed.............................. $\quad .40 \quad 1.25$

Coronata Maxima, yellow.......................................................... .50

Drummondi (Golden Wave) golden yellow, red zone...... $\quad .35 \quad 1.10$

\section{CAMPANULA MEDIUM CALYCANTHEMA (Canterbury}

\section{Bells) Cup and Saucer Varieties}

Dark Blue (violet)

Light Blue (lilac) 2.50

Rose

White

Mixed, extra fine.

CAMPANULA MEDIUM, Single Mixed

CANARY BIRD VINE: See Tropaeolum

\section{CANDYTUFT}

Empress, white

Giant Hyacinth Flowered White, extra select.

This is a very superior strain; it grows with us about $11 / 2$ feet high, with enormous trusses of blooms. It has been one of our specialties for a number of years and every effort is made to keep it at a very high state of perfection. We believe it is superior to any other strain on the market.

Little Prince, a miniature Giant Hyacinth Flowered

White, six inches high, extra select strain 


\section{CANDYTUFT UMBELLATA}

Carmine $\$ 0.40$

Crimson

Flesh Pink

Lavender

Rose Cardinal

White

Waller-Franklin Formula Mixture, "Just a little bit better".

Mixed, all varieties.

CANDYTUFT UMBELLATA HYBRIDA NANA, a dwarf compact group only six inches high.

Crimson.

Flesh

Lilac.

Rose.

White

Choice Mixed.

Perennial types-see Iberis

CANNA, Crozy's Hybrids Choice Mixed

\section{CARNATION}

Chabaud's Everblooming Mixed

Giant Malmaison Mixed.

Grenadin, Cardinal Red

" Snow White

$3.60 \quad 12.00$

" Triumph, pink

$3.60 \quad 12.00$

Marguerite Fine Mixed.

1.50

5.00

\section{CARNATION CHABAUD'S GIANT IMPROVED}

A new acquisition in the line of Carnations; coming into bloom five months from seed and continuing to bloom throughout the Summer. The plants are of robust upright habit; they form ten to twenty stalks, bearing huge flowers of fine form; they come about $90 \%$ double, are quite true and are highly clove scented.

This group should not be confused with the old Chabaud Everblooming.

\begin{tabular}{|c|c|c|c|}
\hline Cardinal Red (Sparkler & $\begin{array}{c}\text { Per oz } \\
\ldots \$ 2.00\end{array}$ & 6.00 & 20.00 \\
\hline imson (Nero).......... & 2.00 & 6.00 & 20.00 \\
\hline se & ... 2.00 & 6.00 & 20.00 \\
\hline & .... 2.00 & 6.00 & 20.00 \\
\hline
\end{tabular}




\section{CARNATION CHABAUD'S GIANT IMPROVED-Continued}

\begin{tabular}{|c|c|c|}
\hline $\begin{array}{c}\text { Per oz. } \\
\ldots \$ 2.00\end{array}$ & $\$ 6.00$ & $\$ 20.00$ \\
\hline e (Legion of Honor) & 6.00 & 20.00 \\
\hline (Jeanne Dionis)................................................... 2.00 & 6.00 & 20.00 \\
\hline (Marie Chabaud) $\ldots \ldots$ & 6.00 & 20.00 \\
\hline .... 1.00 & 3.00 & 10.0 \\
\hline
\end{tabular}

\section{CARNATION CHABAUD'S ENFANT DE NICE}

Carnation Enfant de Nice is the result of a cross between Carnation Chabaud's Giant Improved and Carnation Perpetual. The plants are of robust, upright habit, with long, strong stalks bearing flowers $2 \frac{1}{2}$ to 3 inches in diameter. This strain is magnificent and is so far, the last word in annual Carnations. The color comes about $75 \%$ true from seed and about $90 \%$ of the plants come double.

\begin{tabular}{|c|c|c|}
\hline 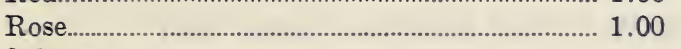 & 3.00 & 10.00 \\
\hline Salmon & 3.00 & 10.00 \\
\hline White. & 3.00 & 10.00 \\
\hline ixed & 1.50 & 5.00 \\
\hline & & er \\
\hline 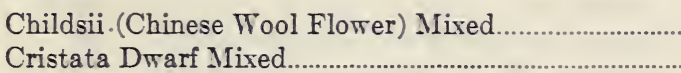 & & \\
\hline 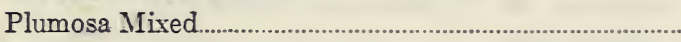 & 0 & 3. \\
\hline
\end{tabular}

CENTAUREA CYANUS: Flora Plena (Cornflower or Bachelor's Button)

Blue (Florists' Strain) rery double........................................ $.40 \quad 1.25$

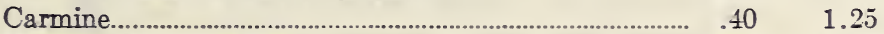

Maroon (Atropurpurea)............................................................ $\quad .40 \quad 1.25$

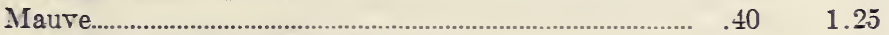

Rose (Taller-Franklin) (Florists' Strain) ...................................40 $\quad .40$

White, very double ....................................................................... $.40 \quad 1.25$

Mixed Double, all rarieties, extra fine................................ $\quad .30 \quad 1.00$

CENTAUREA IMPERIALIS (Imperial Sweet Sultan)

Amaranth Red (Waller-Franklin).......................................... 1.50 5.00

Brilliant Rose..................................................................... $\quad .90 \quad 3.00$

Deep Lavender ………………………………………......... $\quad .90 \quad 3.00$

Delicate Lilac............................................................................. $\quad .90 \quad 3.00$

Purple

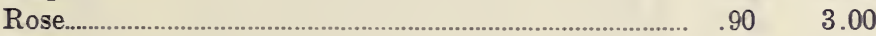

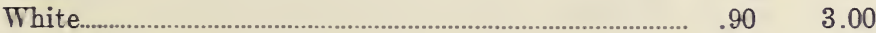

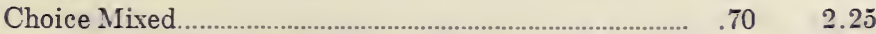


CENTAUREA CANDIDISSIMA (Argentea Vera) silvery leaves.

CENTAUREA GYMNOCARPA (Dusty Miller) silvery leaves.

CENTAUREA SUAVEOLENS, yellow.

CERASTIUM TOMENTOSUM (Snow in Summer) silvery white foliage, rock plant.

CHEIRANTHUS ALLIONI, orange Siberian Wallflower...... . .50

\section{CHELONE BARBATA}

Coccinea, scarlet.................................................................... .75

Hybrida Mixed, shades of pink, rose, carmine, violet.... . .75

CHRYSANTHEMUM SINGLE ANNUAL (Painted Daisy)

Carinatum A trococcineum (The Sultan) dark copper, yellow zone.

" Burridgeanum, white, scarlet zone.

" Chameleon (Eclipse) golden chamois, scarlet zone.

Northern Star, giant white, yellow zone......

" Purpureum (W.E. Gladstone) deep purple, yellow zone.

Segetum Eastern Star, primrose, brown disc................... . .30

" Evening Star (Helios) golden yellow............... $\quad .30 \quad 1.00$

" Gloria Alba, white, pale disc.............................. $\quad .30 \quad 1.00$

" Morning Star, pale primrose ................................. . .30 $\quad 1.00$

" Mixed

\section{CHRYSANTHEMUM DOUBLE ANNUAL}

Carinatum Dunnett's White.

Coronarium Lemon

White. 


\section{CHRYSANTHEMUM LEUCANTHEMUM PERENNIAL}

These Ox-Eye Daisies or Marguerites are all Shasta Daisy Type. After careful trials of all existing varieties we have selected the following group as being the best and they will give a succession of blooms in California from April to October. Dates of blooming are for California.

Elder Daisy, a popular variety for early cut flowers coming into bloom four to five weeks earlier than Shasta Alaska. However, the blooms are smaller with more of them to a plant. In bloom April 10th, height two feet...............................................................\$1. $\$ 1.00$

King Edward VII, very robust large flowers. In bloom June 12th, height two and a half feet.

May Queen, an abundance of medium-sized flowers. In bloom April 15th, height two feet.

Mrs. C. Lowthian Bell, a splendid variety, flowers large white on long stems. In bloom June 12th and continues until October, height three feet.

Perfection, large white flowers, small yellow centers. In bloom June 12th, height two and a half feet.

Shasta Alaska, the most popular of all the Shastas, especially with florists. Very large pure white flowers with small yellow centers. In bloom May 20th. Height two and a half feet. A very shy seeder.

Shasta Westralia, a very fine Shasta Daisy similar to Alaska only of higher growth and blooming slightly earlier. In bloom May 6th, height three feet

\section{CINERARIA MARITIMA}

Candidissima, white leaved................................................... .25

Diamond, white leaved........................................................ . 30

COBAEA (Cathedral Bells)

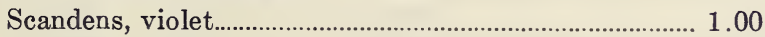

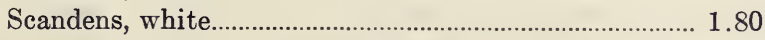

COIX LACHRYMAE (Job's Tears) ....................................... .10

\section{COREOPSIS}

Grandiflora Auricula Superba (New), a serrated band of brownish red encircles the golden disc, flower petals golden yellow

“ Flora Plena (New) golden yellow, semidouble flowers. 
COSMIDIUM Burridgeanum, velvety brown with golden

yellow border.

COSMOS EARLY EXPRESS PINK, an extra early Cosmos

flowering in from forty-five to fifty days from the time seed is planted. The plant grows about two and onehalf to three feet high and is covered with medium sized bright pink flowers. (New) (Waller-Franklin).....

\section{COSMOS EXTRA EARLY DOUBLE CRESTED}

This beautiful group of Double Cosmos is variable as to percentage of doubleness, which does not often exceed fifty per cent. Both double and single flowers will appear on the same plant.

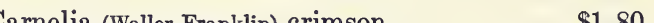

Per oz.

Carnelia (Waller-Franklin) crimson.

Peach Blossom (Waller-Franklin) pink

Whirlwind (Waller-Franklin) white 1.80

Choice Mixed 1.20

\section{COSMOS EXTRA EARLY MAMMOTH FLOWERING}

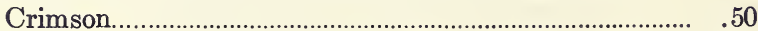

Pink.

White.

Choice Mixed

COSMOS LATE DOUBLE CRESTED

Crimson King.

Pink Beauty

White Queen.

Mixed.

\section{COSMOS LATE SINGLE}

Lady Lenox Giant Flowering Pink ...................................... $\quad .30 \quad 1.00$

" " " " White.................................... .30 . 1.00

Perfection Giant Flowering Crimson.................................... $\quad .30 \quad 1.00$

" " " $"$ Crimson Ray........................... $.30 \quad 1.00$

" " "

Hybrida, Klondyke, orange.................................................. $1.80 \quad 6.00$

Miniature Yellow (Bidens Humulis),.................................. $\quad .30 \quad 1.00$

CYNOGLOSSUM Amabile (Chinese Forget-me-not) brilliant deep ultramarine blue. 
DELPHINIUM CHINENSIS GRANDIFLORUM : Tall (3 feet high)

Dark blue $\$ 0.90 \$ 3.00$

White. .90

Mixed

DELPHINIUM CHINENSIS GRANDIFLORUM : Dwarf (1 foot high)

Azure Fairy, azure blue. 1.80

Blue Butterfly Improved, ultramarine blue.

1.80

\section{DELPHINIUM : Perennial}

Belladonna Improved (Cliveden Beauty) light blue, larger flowered and a stronger grower than the original Belladonna........................................................... 9.00

Belladonna (True) light blue.............................................. 4.50

15.00

Bellamosum, dark blue. 4.50

15.00

Cardinale, scarlet. 6.00

20.00

Gold Medal Hybrids Mixed.

2.50

8.00

Hybrids XXX Art Shades (Waller-Franklin)

$6.00 \quad 20.00$

" XXX Deep Blue Shades (Waller-Franklin)............ 6.00

20.00

" XXX Light Blue Shades (Waller-Franklin).......... 6.00

20.00

“ XXX Mid-Blue Shades (Waller-Franklin),.............. 6.00

20.00

" XXX Mixed (Waller-Franklin).

$4.50 \quad 15.00$

The above is an especially fine group of Delphiniums which we have divided into their various color shades. They contain both single and double flowers and many Wrexham types.

Waller-Franklin Hollyhock Strain Mixed (Wrexham)..12.00 40.00

\section{DIANTHUS HEDDEWIGII (Japanese Pink)}

Crimson Belle, single, deep crimson.................................. $\quad .90 \quad 3.00$

Dark Purple Fl. Pl. (Violet Queen).................................... $1.50 \quad 5.00$

Diadematus Fl. Pl. (Diadem Pink) double mixed........ $\quad .75 \quad 2.50$

Eastern Queen, single rose................................................... $\quad .60 \quad 2.00$

Fireball Fl. Pl., deep scarlet.............................................. $1.25 \quad 4.00$

Mourning Cloak Fl. Pl., black purple, white margin...... $\quad .90 \quad 3.00$

Nobilis Giant Mixed (Royal Pinks) single mixed............ $\quad .60 \quad 2.00$

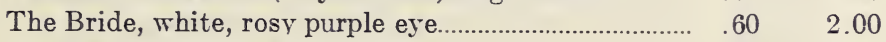

Heddewigii Hybridus double mixed ……………………... $\quad .60 \quad 2.00$

Heddewigii single mixed........................................................ $\quad .60 \quad 2.00$

\section{DIANTHUS HEDDEWIGII LACINIATUS}

Lucifer Fl. Pl., orange scarlet.......................................... $1.25 \quad 4.00$

Mirabilis, single, deeply laciniated petals....................... $\quad .75 \quad 2.50$

Punctatus Mixed (Princess Pink) single............................ $\quad .75 \quad 2.50$ 


\section{DIANTHUS HEDDEWIGII LACINIATUS-Continued}

Salmoneus, Salmon Queen, single salmon.......................\$1.25

$\$ 4.00$

Fl. Pl. Salmon King.

Snowball, Fl. Pl., large white.

Snowdrift, Fl. Pl., pure white.

Vesuvius, single orange scarlet

Heddewigii Laciniated Double Mixed.

Heddewigii Laciniated Single Mixed

\section{DIANTHUS-PINKS VARIOUS}

Caesius (Cheddar Pink) pink, for borders and rock gardens.

Deltoides (Maiden Pink) rose.

Chinensis Double Mixed (Indian Pink)

" Single Mixed.

$.35 \quad 1.10$

Hybridus Latifolius Atrococcineus Fl. Pl., scarlet........

Imperialis Fl. Pl., Imperial Pinks Mixed

Plumarius Double and Semi-double mixed

$\begin{array}{ll}1.80 & 6.00\end{array}$

Single Pink fine mixed.

“

6

Scoticus Double, Scotch Mixed.

$1 / 16 \mathrm{oz}, 1 / 4 \mathrm{oz}$. Per oz.

Perpetual Large Flowered Double Mixed.

6

Large Flowering Double Choice Mixed, sweet scented.

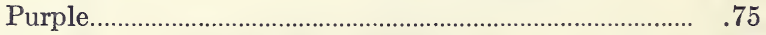

Rose.

White.

Mixed

\section{DIMORPHOTHECA (Star of the Veldt)}

Aurantiaca, Orange Yellow.

Eklonis, pure white with blue disc, long stems. 


\section{DOLICHOS (Hyacinth Bean)}

Lablab White $\$ 0.15$

$\$ 0.40$

“ Princess Helene (Daylight).................................... $\quad .20$

Purple (Darkness) ...................................................................... .15

Soudanensis........................................................................... .30

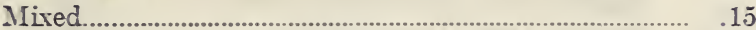

Lignosus, Australian Pea Vine.

\section{ESCHSCHOLTZIA CALIFORNICA (California Poppy)}

Alba

Alba Fl. Pl., double white.

Aurantiaca, rich orange, true California Poppy............. $\quad .30$

Ballet Girl (Waller-Franklin) carmine, creamy white......... 1.50

Canaliculata Crocea, orange fluted.

5.00

Carmine King, deep carmine.

Chrome Queen, amber vellow.

Crocea, Fl. Pl. orange.

Fringed Yellow, buttercup yellow, fringed petals..

Golden West (Californica) yellow with orange center....

Lovely (Waller-Franklin) rose pink suffused salmon.

Mandarin, inside petals orange, outside scarlet...

Mauve Beauty (Waller-Franklin) pure maure

Orange Flame (Waller-Franklin) vivid orange scarlet....... 1.50

Double Mixed, yellow and white only.

Brilliant Mixed, rich in shades of crimson, pink and orange-scarlet.

Hybrida Mixture (Waller-Franklin). New large flowering erect type containing a large percentage of new colors in Eschscholtzias and includes soft pink, scarlet, chrome, copper red, claret and royal purple.

Mixed, white, yellow and orange shades.

\section{EUPHORBIA}

Heterophylla (Mexican Fire Plant) or Annual Poinsettia, carmine.. 


\section{GAILLARDIA (Blanket Flower)}

Picta Single Alba Marginata, brownish red with white margin $\$ 0.60$ $\$ 2.00$

Chameleon, white, yellow and scarlet..... $\quad .60$

Indian Chief, bronzy red.

Yellow

Picta Lorenziana Double Bicolor, red and yellow........ $\quad .75 \quad 2.50$

$\begin{array}{ll}\text { " } & \\ 4 & 4 \\ \text { " } & 4\end{array}$

" Canary Yellow, very large

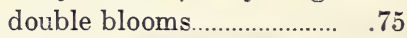

Grandiflora Dazzler, golden yellow and maroon red....... 3.00 shades of bronzy red, golden tipped, very robust.

GERBERA (Transvaal Daisy) Jamesoni Hybrids, per 1,000 Seeds, \$2.50.

\section{GEUM}

Lady Stratheden, double orange yellow

Mrs. Bradshaw, double scarlet.

\section{GILIA}

Capitata (Queen Ann's Thimble) lavender blue............ . .25

Tricolor Mixed.

GLAUCIUM (Horned Poppy) orange scarlet

GODETIA TALL DOUBLE GRANDIFLORA-Produces

fine gladiola-like flowers in long trusses; excellent for cutting.

Carmine, soft pink, carmine blotch................................... $.75 \quad 2.50$

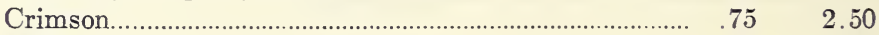

Mauve

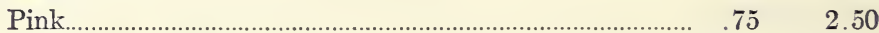

Rosy Morn, bright pink ....................................................... $\quad .75 \quad 2.50$

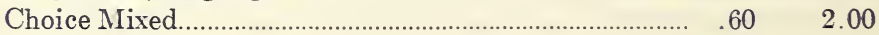




\section{GYPSOPHILA ELEGANS}

Elegans alba. $\$ 0.15 \$ 0.40$

Grandiflora Alba, Paris Market, extra select. .15 .50

\section{GYPSOPHILA PANICULATA}

Per oz.

Double Snow White. $\$ 3.00 \quad 9.00$

30.00

Single, White. 2.00

\section{HELIANTHUS: See Sunflower}

HELICHRYSUM MONSTROSUM : Flora Plena (Strawflower)

Canary Yellow (Waller-Franklin) (Buttercup)..................... . .90

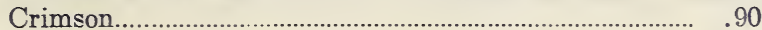

Fireball

Golden Globe

3.00

Rose Carmine Shades.

Rose Queen (Waller-Franklin) rich deep rose....................... . .90

Salmon

Salmony Red

Scarlet.

Silvery Pink (New) (Waller-Franklin) silvery cameo pink. .90

Violet.

White

Mixed, extra fine.

\section{HOLLYHOCK}

Chater's Double Chamois.

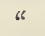

Deep Rose.

Maroon

Newport Pink.

Salmon Rose.

Scarlet.

Sulphur Yellow.

White.

Superb Mixed

Alameda Pink, light pink shades, Allegheny type.

Imperator, very large fringed flowers 6 inches across with double rosette in center, colors ranging through delicate pink to cerise salmon.

Allegheny Mixed, semi-double, pastel shades.

Single Chamois

Maure.

Pink.

Rose 


\section{HOLIYHOCK-Continued}

Single Scarlet

White.

" Yellow

" Mixed

HUNNEMANNIA (Mexican Tulip Poppy) Fumariaefolia.... .75

IBERIS (Perennial Candytuft) Per oz.

Gibraltarica, lilac shading to white 1.50

Queen of Italy, rosy lilac.

Sempervirens, perennial white.

KOCHIA (Summer Cypress) Trichophylla Childsii.

LARKSPUR: Emperor Dwarf Branching Double Stocix Flowered, about twenty-four inches high.

Ageratum Blue.

Brilliant Carmine (New) (Waller-Franklin) identical in color to Lustrous Carmine in the Tall Branching Group.

Mauve Rose.

Pink Delight (Waller-Franklin).

Violet Purple.

White

Choice Mixed

LARKSPUR: Early Giant Hyacinth Flowered Improved, about thirty-six inches high, early flowering.

Dark Blue.

Lilac

Pink

Purple

White.

Choice Mixed

LARKSPUR: Tall Branching Double Stock Flowered.

Bright Rose, is mauve rose............................................... . .60

Dark Blue.

Exquisite Pink (Variable) (Waller-Franklin)

Exquisite Pink Improved (New) (Waller-Franklin) soft pink shaded salmon, Award of Merit R. H. S.

Exquisite Rose (New) (Waller-Franklin) rose pink, Award of Merit R. H. S. 
LARKSPUR: Tall Branching Double Stock Flowered-Continued

Lilac.

Lustrous Carmine.

Rosy Scarlet.

Shell Pink.

Sky Blue.

White Spire, dazzling pure white, upright Delphinium habit (New) (Waller-Franklin) see page 5 .

White..

Waller-Franklin Formula Mixture, "Just a little bit better".

Mixed.

LARKSPUR: Tall Branching Single.

Blue .40

Mauve.

Pink Pearl, salmon pink.

Rosy Pink.

White.

Mixed

\section{LATHYRUS LATIFOLIUS (Everlasting Pea)}

Pink Beauty.

Red.

White Pearl.

Mixed.

\section{LAVATERA (Mallow)}

Assurgentiflora (California Windbreak) rose................. $\quad .30$

Splendens Rosea $\$ 0.60 \$ 2.00$

$\begin{array}{ll}.75 & 2.50\end{array}$

$.75 \quad 2.50$

$.60 \quad 2.00$

$.60 \quad 2.00$

$3.00 \quad 10.00$

$.60 \quad 2.00$

$.60 \quad 2.00$

$.50 \quad 1.50$

" Sunset,

White.

LEPTOSYNE Maritima Grandiflora, yellow.

\section{LINUM}

Per oz.

Grandiflorum Rubrum (Scarlet Flax).

Narbonense, steel blue

Perenne, Sibiricum, blue.

LUNARIA Biennis (Honesty) white. 


\section{LUPINUS}

Hartwegii Albus. $\$ 0.30 \$ 1.00$

" Dark Blue, Oxford Blue "Highly Commended" R. H. S........................................... $\quad .30 \quad 1.00$

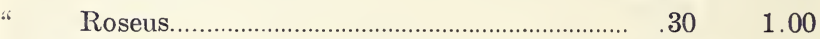

6 Sky Blue, Cambridge Blue............................... $\quad .30 \quad 1.00$

" Mixed............................................................... . .25

Hybridus Roseus, pink ……………………………………..... $\quad .60 \quad 2.00$

Nanus Blue...................................................................... $\quad .50 \quad 1.50$

\section{MARIGOLD TALL AFRICAN DOUBLE FISTULOSA}

An especially fine tall branching strain, bearing enormous flowers of the quilled type.

Lemon Queen........................................................................ $1.50 \quad 5.00$

Orange Prince...................................................................... $1.50 \quad 5.00$

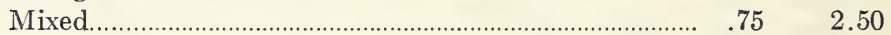

\section{MARIGOLD TALL FRENCH DOUBLE}

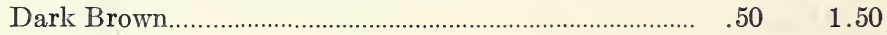

Lemon

Orange

Scotch Gold Striped............................................................... $1.50 \quad 5.00$

Spotted (Pulchra Plena) yellow, spotted brown............ $\quad .50 \quad 1.50$

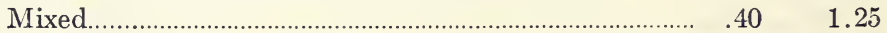

Australian Giant or Grendon Tree, brownish yellow,

very tall, with hardly any marigold odor..................... $\quad .90 \quad 3.00$

Single Brown (Josephine) for Florists.................................. $\quad .50 \quad 1.50$

\section{MARIGOLD DWARF FRENCH DOUBLE}

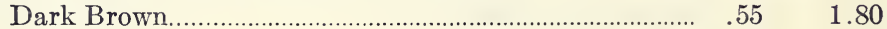

Golden Ball, very compact, large golden yellow............ $\quad .55 \quad 1.80$

Golden Yellow Brown Margin, large flowering............... $\quad .55 \quad 1.80$

Pallida, dwarf compact, pale sulphur yellow................... $\quad .55 \quad 1.80$

Robert Beist, dark maroon................................................ $\quad .60 \quad 2.00$

Spotted (Pulchra Nana) yellow, spotted brown............ $\quad .55 \quad 1.80$

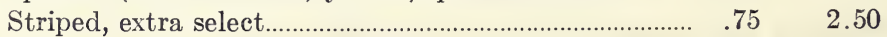

Mixed, extra choice............................................................... $\quad .50 \quad 1.50$

\section{MARIGOLD DWARF FRENCH SINGLE}

Diadem, mahogany red, edged gold.................................. $\quad .40 \quad 1.25$

Fire Cross, saffron orange with brown red center.......... $\quad .75 \quad 2.50$

Gold Star, yellow striped maroon......................................... $\quad .40 \quad 1.25$

Legion of Honor (Little Brownie) .................................... $\quad .50 \quad 1.50$ 
MARIGOLD DWARF FRENCH SINGLE-Continued

Silver King, pale yellow, maroon at base ………….....\$0.40

Star of India, crimson striped yellow................................ $\quad .40$

Tagetes Signata Pumila, very dwarf orange.................... 1.50

5.00

Mixed.

\section{MATRICARIA}

Capensis Alba Plenissima

Eximia Nana Fl. Pl. Golden Ball.

\section{MIGNONETTE (Reseda)}

Reseda Odorata Grandiflora Improred …………........ . .15

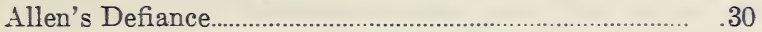

Golden Queen, compact bedding, yellow ………………..... $\quad .30 \quad 1.00$

Machet.

" Bismarck, extra large spikes

Giant Flowering, selected strain ........................... $\quad .90 \quad 3.00$

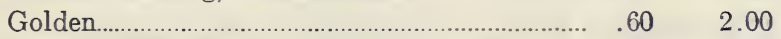

Golden Goliath, giant golden yellow.................. $1.50 \quad 5.00$

Goliath Red, large intense red spikes................ $1.25 \quad 4.00$

New York Market, Florists' strain................... 1.50 5.00

Orange Queen............................................................... $\quad .60 \quad 2.00$

White, carefully selected strain ........................... $1.25 \quad 4.00$

4. Special Mixture..................................................... $\quad .30 \quad 1.00$

Dobbie's Giant, mammoth red spikes.............................. $\quad .40 \quad 1.25$

Gigantea Pyramidalis, giant red spikes............................... $\quad .40 \quad 1.25$

Salmon Red (Salmon Queen)........................................... $\quad .50 \quad 1.50$

Victoria Perfecta, brilliant red spikes............................... $\quad .50 \quad 1.50$

MINA Lobata (Flag of Spain) scarlet and orange

climber

Per oz.

MIRABILIS (Four O'Clocks) Marvel of Peru Mixed..

\section{NASTURTIUM DWARF (Tropaeolum)}

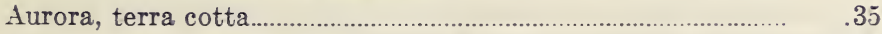

Beauty, yellow and scarlet........................................................ .35

Bronze, reddish golden bronze.................................................. .35

Chameleon, choice mixture of rare colors....................................

Cloth of Gold, fiery red, golden leared...................................

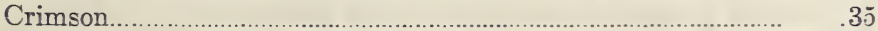

Crystal Palace Gem, sunflower yellow, spotted purple maroon $\quad .35$

Empress of India, dark scarlet, dark leaved................................. . .35 


\section{NASTURTIUM DWARF-Continued}

Golden King, Indian yellow, dark leaved.................................... \$0.3

" Queen, bright yellow, golden leaved.................................. . .35

Ivy Leared Mixed, laciniated.......................................................... . .35

King of Tom Thumbs, bright scarlet, dark leaved...................... . . .35

" Theodore, purple-garnet, dark leaved.................................. . .35

Pearl or Moonlight, amber white..................................................... . .35

Prince Henry, pale yellow, spotted carmine red......................... . .35

Queen of Tom Thumbs, variegated leaved mixed....................... $\quad .35$

Regelianum, purple-garnet................................................................... . .35

Roseum Coeruleum............................................................................ .35

Ruby King, bluish rose..................................................................... . .35

Spencer Choice Mixed, waved leaves............................................... . . 35

Spotted King or Ladybird, yellow, spotted red........................... . . .35

Vesuvius or Salmon Queen, salmon rose............................................ . .35

Yellow............................................................................................. .35

Special Fancy Mixture of Dark Leaved Varieties........................ . .35

Fine Dwarf Mixed................................................................................

\section{NASTURTIUM TALL or TRAILING, Including Lobb's.}

Owing to the similarity in color and type among the Tall and Lobbianum Nasturtiums we have taken the best in each group and combined them under the heading of Nasturtium Tall or Trailing.

Brilliant or Fulgens (Lucifer) bright scarlet, dark leaved........ \$0.35

Butterfly, sunflower yellow spotted salmon pink........................ . .35

Chameleon (Coquette) choice mixture of rare colors................ . . .35

Cloth of Gold, fiery red........................................................................ .35

Crimson, velvety crimson................................................................... .35

Dunnett's Orange (Sunlight).......................................................... .35

Edward Otto, lilac brown................................................................ .35

Firefly, yellow, spotted red.................................................................. .35

Fordhook Fashion, terra cotta on yellow ground........................ . .35

Giant of Battles, amber yellow spotted bright red.................... . . .35

Gold Garnet, orange yellow, spotted garnet..................................

Golden Queen, sunflower yellow........................................................ . .35

Hemisphericum, bright yellow, marbled salmon rose................. $\quad .35$

Ivy Leaved Mixed, laciniated............................................................ . .35

Jupiter, golden yellow large flowered.............................................. . .35

King of the Blacks, dark purple....................................................... $\quad .35$

King Theodore (Black Prince) purple-garnet, dark leaved...... . . .35

Madam Gunther's Hybrids, variety of brilliant colors............... . .35

Pearl or Moonlight (Asa Gray) amber white................................ . . .35

Prince Henry, amber yellow, spotted carmine red...................... . .35

Regelianum, purplish crimson........................................................... . .35 


\section{NASTURTUIM TALL or TRAILING-Continued}

Rose

Ruby (Rudolph Virchow) bluish rose..

Scarlet (Spitfire) rosy scarlet.

Schulzi, scarlet dark leaved; darker than Brilliant or Fulgens and the nearest to Empress of India.

Shillingi, yellow, spotted maroon....................................................... .35

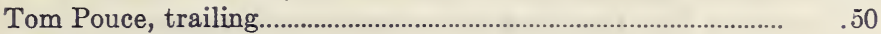

Twilight, delicate salmon ………………………………………........ .35

Variegated Mixed (Lobb's Queen Type).......................................... . .35

Vesuvius or Salmon Queen (Regina) salmon rose......................... . .35

Yellow, sunfiower yellow.................................................................. .35

- California Giants Special Mixture.....................................................

California Giants Mixed................................................................... $\quad .30$

NEMESIA

Price Price

Strumosa Grandiflora Suttoni, Orange

Per oz. $1 / 4 \mathrm{lb}$.

Strumo

"

Nana Compacta Blue Gem Improved

Triumph Mixed.
$\$ 2.50 \quad \$ 7.50$

$2.50 \quad 7.50$

$2.50 \quad 7.50$

$2.00 \quad 6.00$

$2.50 \quad 7.50$

3.00

9.00

\section{NEMOPHILA}

Insignis Blue (Baby Blue Eyes) .......................................... $\quad .30 \quad 1.00$

Choice Mixed, all sorts............................................................. $\quad .30 \quad 1.00$

\section{NICOTIANA}

Affinis, Sweet Scented White

" Hybrida Grandiflora Mixed, sweet scented.. 60

Sanderae, Crimson King, dark velvety crimson red..... $\quad .90$

\section{PENTSTEMON}

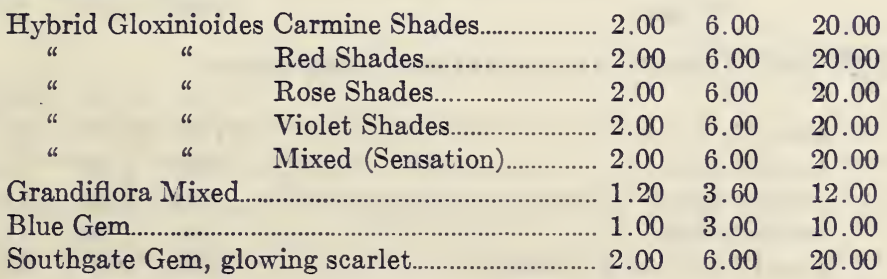




\section{PETUNIA HYBRIDA}

Alba, pure white. $\$ 1.50$

$\$ 5.00$

Carmen Sylva (Baby Blue) violet, white throat........... 1.50

Countess of Ellesmere, rose, white throat.

General Dodds, velvety blood red.

Golden Rose (Lord Courtenay)

Howard's Star, purple, white star.

Howard's Star Improved (Waller-Franklin) velvety purple, pure white star.

Inimitable, blotched and striped.

Violacea, deep violet

De Luxe Bedding Mixture (Waller-Franklin). A mixture made up of large flowering hybrid sorts...

Waller-Franklin Formula Mixture.

Mixed, excellent mixture of small flowered sorts......... 1.25

Nana Erecta Alderman, deep violet.

$10: 00$

"

" " Inimitable, dwarf blotched and striped....

" " Norma, blue with white star.

3.00

2.75

2.50

10.00

9.00

8.00

12.00

Rose of Heaven, dwarf, rose pink.

3.60

10.00

15.00

12.00

Senator, purple with white throat.

3.00

4.50

3.60

Silver Blue, light blue.

6.00

Snowball, pure white, very compact........ 3.00

20.00

10.00

Snow Queen, white, compact.

3.60

Violacea, deep violet, the finest strain of violet Petunia on the market...

Mixed, all Nana Erecta varieties, fine.

2.50

15.00

8.00

\section{PETUNIA HYBRIDA PENDULA (BALCONY PETUNIA)}

Per oz.

\begin{tabular}{|c|c|c|}
\hline & 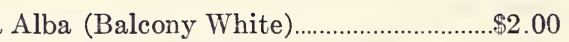 & 6.00 \\
\hline “ & Purpurea (Balcony Crimson)................. 2.00 & 6.00 \\
\hline " & Rose) ............ & \\
\hline “ & Improved (Balcony Blue)...... 3 & \\
\hline " & Mixed. & 6.00 \\
\hline
\end{tabular}

\section{PETUNIA HYBRIDA GRANDIFLORA, Plain Edged.}

$\frac{1}{16}$ oz. $1 / 4$ oz. Per oz.

Alba $\$ 1.60 \quad \$ 4.80 \quad \$ 16.00$

Aurora, pink

$1.60 \quad 4.80$

16.00

Balcony Queen (Admiration) violet and white.. 1.60

4.80

16.00

Brilliant Rose.

$1.60 \quad 4.80$

16.00

Dwarf Pink Glory (Diener's)

$\begin{array}{ll}3.00 & 9.00\end{array}$

30.00 


\section{PETUNIA HYBRIDA GRANDIFLORA, Plain Edged-Continued}

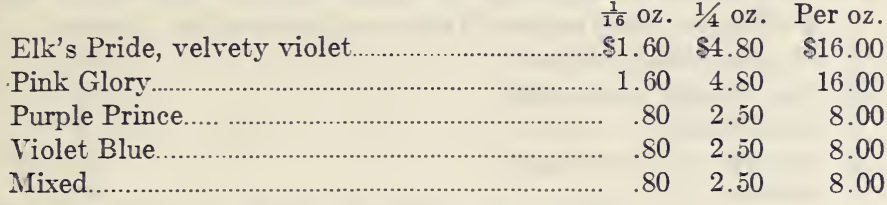

\section{PETUNIA HYBRIDA GRANDIFLORA FIMBRIATA}

\begin{tabular}{|c|c|c|}
\hline Brilliant Rose..... & 1.60 & 4.80 \\
\hline Miranda, rosy carmine & 1.60 & 4.80 \\
\hline Portland Beauty, crimson, maroon throat....... & 1.60 & 4.80 \\
\hline Pride of Portland, rose pink & 1.60 & 4.80 \\
\hline Romany Lass, coppery crimson, dark throat... & 4.50 & 13.50 \\
\hline Scarlet Beauty.................... & 1.60 & 4.80 \\
\hline Theodosia, rosy pink with golden center... & 2.50 & 7.50 \\
\hline Triumph Pink............... & 1.60 & 4.80 \\
\hline White Beauty...................... & 1.60 & 4.80 \\
\hline Fimbriata Single Mixed... & 1.60 & 4.80 \\
\hline
\end{tabular}

\section{PETUNIA FLUFFY RUFFLES}

Choice mixed, special strain................. $4.80 \quad 16.00$

PETUNIA GIANTS OF CALIFORNIA. The largest

and finest of all single Petunias.

Choice mixed, special strain................................................ $5.40 \quad 18.00$

\section{PETUNIA DWARF GIANTS OF CALIFORNIA}

Choice Mixed

\section{PETUNIA GIANT DOUBLE FRINGED}

Grandiflora Flora Plena Fimbriata Mixed. A special strain of Double Fringed Petunia producing a very high percentage of extremely large full double flowers. We confidently believe this strain excels any other strain on the market.

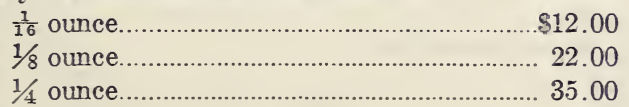

PHACELIA

Price Price $1 / 4 \mathrm{lb}$. per lb.

Campanularia (California Blue Bell) gentian blue.........\$1.00 $\$ 3.50$

Tanacetifolia, light blue (for bees) ..................................... . .15 $\quad .50$

PHLOX DRUMMONDI, Grandiflora Mixed......................... $2.10 \quad 7.00$

PHYSOSTEGIA VIRGINICA (Obedient Plant) pink ............ $3.60 \quad 12.00$ 


\section{POPPY SHIRLEY DOUBLE}

Art Shades (New) colors of Indian and carmine lake...\$1.50 $\$ 5.00$

Eldorado Double Mixed....................................................... $\quad .60$

2.00

Pink Shades, extra select......................................................... $\quad .60 \quad 2.00$

Queen Mixed............................................................................. $\quad .60 \quad 2.00$

Ryburgh Hybrids Mixed......................................................... $\quad .60 \quad 2.00$

Salmon.

\section{POPPY SHIRLEY SINGLE}

American Legion (Waller-Franklin) brilliant orange scarlet, Highly Commended, R. H. S................................... .40

Blue Shades, grey blue......................................................... 1.00

3.50

Deep Apricot

Glaucum (Tulip Poppy) intense scarlet............................ $\quad .60$

2.00

Picotee, white cup, scarlet edging........................................ . .60

2.00

Salmon Pink.

White.

Wild Rose Pink.

Waller-Franklin Formula Mixture, "Just a little bit better" containing both Single and Double flowers.... .40

\section{POPPY SINGLE ANNUAL}

Admiral, pure white, edged scarlet..................................... .20

Dainty Lady, heliotrope, violet center............................ .20

King Edward, scarlet, shaded crimson................................. . .20

Miss Sherwood, white, edged rose...................................... $\quad .20$

The Bride, pure white, fringed............................................. $\quad .20$

Virginia, pure white, edged soft pink................................. $\quad .20$

POPPY TALL CARNATION FLOWERED DOUBLE (Fringed Petals)

Bright Rose............................................................................ . . .30 1.00

Fairy Blush Hybrids, mixture of light pink shades..... $\quad .30 \quad 1.00$

Heliotrope........................................................................... $.30 \quad 1.00$

Mikado, red, white striped................................................... $\quad .30 \quad 1.00$

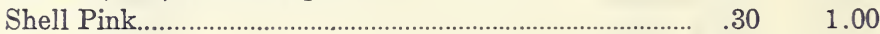

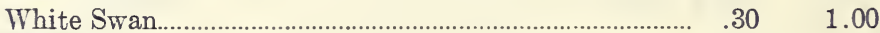

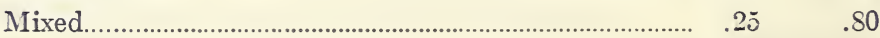

POPPY : Double mixed all varieties............................................... $\quad .25 \quad .80$

POPPY TALL PAEONY FLOWERED DOUBLE (Plain Petals)

American Flag, white, margined scarlet.......................... $\quad .30 \quad 1.00$

Bright Rose ......................................................................... $\quad .30 \quad 1.00$ 
POPPY TALL PAEONY FLOWERED DOUBLE (Plain Petals)

\section{-Continued}

Cardinal Red. $\$ 0.30$

Creamy Yellow 1.00

Shrimp Pink .30

White 1.00

Mixed

PORTULACA GRANDIFLORA Single Mixed

Parthenifolium Aureum (Golden Feather).

2.00

Uliginosum, white.

\section{RICINUS (Castor Oil Plant)}

Sanguineus, red stalks..................................................... .20

Zanzibariensis Mixed ........................................................... .25

ROMNEYA Coulteri (Matilija Poppy) white.

RUDBECKIA Bicolor Superba golden yellow, dark spots.. .75

SALPIGLOSSIS EMPEROR (Painted Tongue)

Alba Lutea (White and Gold).......................................... 1.50

Brown and Gold.

Chamois

Crimson.

Faust, black

Light Blue and Gold.

Primrose.

Purple and Gold

Rose and Gold.

Scarlet and Gold

Velvety Red.

Velvety Violet

Violet and Gold.

\section{SALVIA}

Azurea Grandiflora, blue

Farinacea, light blue. 
SAPONARIA Vaccaria

Rose

White.

SCABIOSA: Grandiflora Flora Plena

Ageratum Blue

Azure Fairy (Azure Blue)

Crimson

Fire King, rich rosy crimson.

Flesh Pink

King of the Blacks, reddish black

Peach Blossom (Waller-Franklin) peach blossom pink Highly Commended, R. H. S.

Red

Rose

Shasta (Waller-Franklin) pure white, enormous size

Sulphur Yellow

White

Choice Mixed.

\section{SCABIOSA CAUCASICA}

Lavender-blue

White

SCABIOSA COLUMBARIA (Anthemifolia)

Delicate Lavender.

Delicate Pink.

SCHIZANTHUS: Grandifiorus Hybridus

Per oz.

Mauve and Purple Shades 2.50

Rose and Amber Shades.

Rose and Carmine Shades

Rose and Pink Shades.

Waller-Franklin Giant Flowering Hybrids, an extra select hothouse strain containing a perfect blend of colors.

SCHIZANTHUS: Wisetonensis

Brilliant Mixture, containing all the brilliant colors.

Choice Mixed.

$.60 \quad 2.00$

SHASTA DAISIES: See Chrysanthemum Leucanthemum 


\section{SOLANUM}

Capsicastrum Nanum (Jerusalem Cherry) small red

fruit... $\$ 0.90$

$\$ 3.00$

Clevelandi, Cleveland Cherry.

\section{STATICE (Sea Lavender)}

Bonduelli, golden yellow.

Caspia, lilac

Perezii, rich blue.

Sinuata Lavender.

" Rosea Superba

“ True Blue, extra deep.

" White.

.20

" Mixed.

Suworowii (Russian Statice) rose.

\section{STOCKS ARE ONE OF OUR PRINCIPAL SPECIALTIES.}

Our percentage of doubles equals the best European pot grown strains. We received five "Awards of Merit" and two "Highly Commended" for our strains of Ten Week and Perpetual Branching Stocks at the "Trial of Stocks" conducted by The Royal Horticultural Society of Great Britain, at Wisley, England.

\section{STOCK DOUBLE LARGE FLOWERING DWARF TEN WEEK}

Aurora, cream tinted pink. $\$ 1.40 \$ 4.20$

Azure Blue.

$1.40 \quad 4.20$

$\$ 14.00$

Blood Red.

1.40

4.20

14.00

Bright Pink.

1.40

4.20

Brilliant Crimson (Triumph)

1.40

4.20

Brilliant Rose.

1.40

Canary Yellow

1.40

Carmine.

4.20

4.20

14.00

Carmine Pink.

1.40

Coppery Carmine.

1.40

Crimson.

Dark Blue

4.20

4.20

14.00

Deep Cream

Deep Purple

Deep Rose.

Flesh, Award of Merit, R. H. S.

Lavender.

Light Blue.

Old Rose.

1.40

Pansy Violet.

1.40 


\section{STOCK DOUBLE LARGE FLOWERING DWARF TEN} WEEK-Continued

Purple. $\$ 1.40 \$ 4.20$

$\$ 14.00$

Rose 1.40

White, Award of Merit, R. H. S.

$1.40 \quad 4.20$

Waller-Franklin Formula Mixture, "Just a little bit better," bright colors only, made up from named varieties.

Choice Mixed

Mixed.

\section{STOCK DOUBLE WALLFLOWER LEAVED TEN WEEK}

Apple Blossom, flesh. 1.20

Canary Yellow.

$1.20 \quad 3.60$

Rose 1.20

3.60

Scarlet

1.20

3.60

12.00

Snowflake, pure white

1.20

3.60

12.00

Violet Queen

$1.20 \quad 3.60$

12.00

Mixed.

.80

2.50

8.00

STOCK DOUBLE EARLY GIANT IMPERIAL (Improved Bismarck)

An especially meritorious class. Growing from twenty-four to thirty inches tall; of branching habit. Very early blooming and producing a high percentage of doubles.

Antique Copper (New) (Waller-Franklin) rich

Hellebore red.

Blood Red

Blush (Apple Blossom)

2.00

6.00

20.00

Canary Yellow (Golden Ball).

2.00

6.00

20.00

Chamois, ivory, tinted old rose.

2.00

6.00

20.00

Chamois Rose

2.00

Dark Blue

2.00

Elk's Pride (New) (Waller-Franklin) royal purple..

3.50

10.50

35.00

Fiery Blood Red.

Flesh.

Golden Rose (New) (Waller-Franklin) light golden rose

$\begin{array}{rrr}3.50 & 10.50 & 35.00 \\ 2.00 & 6.00 & 20.00 \\ 2.00 & 6.00 & 20.00 \\ 2.00 & 6.00 & 20.00\end{array}$

Lavender.

Mauve Lavender.

White.

Choice Mixed 


\section{STOCK DOUBLE GIANT EXCELSIOR (Improved Column Type)}

Each plant produces one large flowering spike about three feet high.

Crimson $\$ 5.00 \$ 15.00 \$ 50.00$

Light Blue. $5.00 \quad 15.00$

50.00

Purple $5.00 \quad 15.00$ 50.00

Rose. $5.00 \quad 15.00$ 50.00

Silvery Lilac. $5.00 \quad 15.00$ 50.00

White. $5.00 \quad 15.00$ 50.00

Fine Mixed. $\begin{array}{ll}4.50 & 13.50\end{array}$ 45.00

\section{STOCK DOUBLE MAMMOTH BEAUTY OF NICE}

American Beauty, deep rose pink.

1.40

4.20

14.00

Aurora (Chamois) cream tinted pink.

Canary Yellow (Monte Carlo).

Carmine Rose (Abundance).

Crimson (Souv. de Monaco)

Deep Blue (Summer Night)

Flesh Pink (Beauty of Nice).

Light Blue (Côte d' Azure).

Old Rose (Belle de Naples)

Pale Violet (Parma Violet).

Rosy Lilac (Queen Alexandra)

Salmon Rose (Salmon King).

1.40

4.20

14.00

White Shaded Pink (Almond Blossom).

1.40

4.20

14.00

White (Mont Blanc)

4.20

14.00

Choice Mixed.

STOCK DOUBLE PERPETUAL BRANCHING (Giant Perfection)

A fine group, especially for Florists. Very early and free blooming. Produces a high percentage of doubles.

Blood Red (Brilliant)........................................
Canary Yellow (Creole) Award of Merit

R. H. S.

Flesh.

Heatham Beauty, rose shaded terra cotta

Pale Blue (May Queen)

" Blush (La France)

Queen of The Belgians, pale violet.....

Rose, rose pink, Award of Merit, R. H. S.

Silvery Lilac (Empress Augusta Victoria)

Award of Merit, R. H. S..

Snowdrift, pure white.

Violet (Sapphire)

Violet Slate (President Wilson).

White (Princess Alice).

Choice Mixed. $\begin{array}{lll}1.60 & 4.80 & 16.00\end{array}$

$\begin{array}{lll}1.60 & 4.80 & 16.00\end{array}$

$\begin{array}{lll}1.60 & 4.80 & 16.00\end{array}$

$\begin{array}{lll}1.60 & 4.80 & 16.00\end{array}$

$\begin{array}{lll}1.60 & 4.80 & 16.00\end{array}$

$\begin{array}{lll}1.60 & 4.80 & 16.00\end{array}$

$\begin{array}{lll}1.60 & 4.80 & 16.00\end{array}$

$\begin{array}{lll}1.60 & 4.80 & 16.00\end{array}$

$\begin{array}{lll}1.60 & 4.80 & 16.00\end{array}$

$\begin{array}{lll}2.00 & 6.00 & 20.00\end{array}$

$\begin{array}{lll}1.60 & 4.80 & 16.00\end{array}$

$\begin{array}{lll}1.60 & 4.80 & 16.00\end{array}$

$\begin{array}{lll}1.60 & 4.80 & 16.00\end{array}$

$\begin{array}{lll}1.40 & 4.20 & 14.00\end{array}$


STOCK DOUBLE EMPEROR (Large Flowering Winter)

Crimson King, deep crimson.

$\$ 2.50 \quad \$ 7.50$

$\$ 25.00$

Egyptian, rose

$2.50 \quad 7.50$

25.00

Empress Elizabeth, bright carmine.

$3.50 \quad 10.50$

35.00

Violetta (New), dark violet.

$3.50 \quad 10.50$

35.00

White Lady, very double, pure white

$3.50 \quad 10.50$

35.00

Choice Mixed.

$\begin{array}{lll}2.50 & 7.50 & 25.00\end{array}$

\section{SUNFLOWER (Helianthus)}

Intermediate Sanguineus, shades of red, purple and reddish brown... $.50 \quad 1.50$

Lenticularis, tall, yellow... .15

Tall Red. .25 .50

Tall Mixed .15 


\section{EARIY FLOWERING SPENCER SWEET PEAS}

Our list of Earlies is very complete and embodies the BEST of the older varieties and all of the newer sorts.

Our Earlies are grown especially for those of the wholesale trade who cater to the florist growers and our cultures are practically PURE LINE STRAINS.

All White (Waller-Franklin) largest pure white.

Amethyst (Waller-Franklin) royal purple.

Angelus, salmon pink...

Asta Ohn (Waller-Franklin) lavender suffused mauve.

Aviator (Waller-Franklin) dazzling crimson scarlet.......

Ball Blue, clear blue.

Ball Orange, rich orange.

Ball Rose, rose pink.

Blanche Ferry (Waller-Franklin) rose pink, white wings.

Blue Bird, violet blue.

Blue Bonnet (New) (Waller-Franklin) best clear deep blue..

Blue Boy, clear blue.

Blue Picotee (Waller-Franklin) white edged violet blue.

Blue Wonder, clear blue.

Bright Light, flaming scarlet.

Burpee's Lavender, light Wisteria-lavender..

Burpee's Orange, deep orange.

Canary Bird, rich cream..

Charm, rich rose pink

Chevalier, rose

Columbia, salmon rose standard, white wings.

Cream (Waller-Franklin)...

Duplex Gem, soft rose pink.

Duplex Pioneer, rose pink.

Edward Zvolanek, lavender.

Eldorado (Waller-Franklin) orange.................................................... $\quad 3.00$

Embers, orange salmon............................................................... 3.00

Enchantress, rose pink

Fair Maid (Waller-Franklin) blush pink suffused salmon.................. $\quad 3.00$

Flambeau (Waller-Franklin) orange cerise.......................................... $\quad 3.00$

Flamingo, orange salmon............................................................ $\quad 3.00$

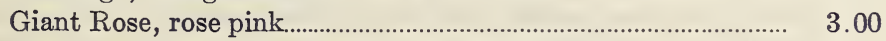

Gilda Gray, salmon cerise overlaid bright pink ......................... $\quad 3.00$

Gleam, bright geranium pink …………………………………….... $\quad 3.00$

Glitters, cerise

Grenadier (Waller-Franklin) dazzling scarlet..................................... $\quad 4.00$

Harmony, clear lavender.......................................................... $\quad 2.50$

Heather Bell, mauve lavender......................................................... $\quad 3.00$ 


\section{EARLY FLOWERING SPENCER VARIETIES-Continued}

Helen Lewis (Waller-Franklin) orange salmon................................. \$ 3.00

Hercules (Waller-Franklin) rosy pink.................................................. $\quad 3.00$

Illumination, salmon cerise........................................................... $\quad 2.50$

Imperial Pink (Waller-Franklin) large clear pink .............................. $\quad 3.00$

Irene (Waller-Franklin) clear lavender.............................................. $\quad 3.00$

Jeanne Mamitsch, rose pink with exceptionally long stems..... $\quad 3.00$

Lavender King, lavender................................................................ $\quad 3.00$

Madonna (Waller-Franklin) giant white, black seeded...................... $\quad 3.00$

Majestic Rose (New) (Waller-Franklin) brilliant rose pink, has vigor and long stems.

Marine, large clear blue................................................................. $\quad 7.00$

Mauve Beauty, rosy mauve............................................................. $\quad 3.00$

Meadow Lark, cream................................................................. $\quad 3.00$

Melody, rose pink ...................................................................... 3.00

Miss Louise Gude, brilliant pink ................................................. $\quad 3.00$

Miss Spokane, cerise-red, scarlet wings........................................... $\quad 3.00$

Monarch, lavender..................................................................... $\quad 3.50$

Morning Star, salmon pink ............................................................ $\quad 3.00$

Mrs. A. A. Skach, deep pink ...................................................... $\quad 3.00$

Mrs. Calvin Coolidge, rich salmon pink ……………................... $\quad 3.00$

Mrs. Charles Zvolanek, lavender................................................... $\quad 3.00$

Mrs. Herbert Hoover, clear blue............................................... 10.00

Mrs. H. R. Holscher, clear pink, robust grower........................ 10.00

Mrs. Kerr, salmon.......................................................................... $\quad 3.00$

New Blue (Waller-Franklin) deep blue................................................. $\quad 3.00$

Orange King (Waller-Franklin) glowing intense orange................... $\quad 4.00$

Oriental, deep cream................................................................... $\quad 7.00$

Othello (Waller-Franklin) maroon...................................................... $\quad 3.00$

Penrose, rose pink ........................................................................ 3.00

Pink Charm (Waller-Franklin) light rose pink.................................. $\quad 5.00$

Pink Cherokee (Waller-Franklin) pink suffused salmon................... $\quad 3.00$

Pink Profusion, rich pink......................................................... $\quad 3.00$

Princess, lavender suffused mauve................................................. $\quad 3.00$

Queen Crimson, ox-blood crimson................................................ $\quad 3.00$

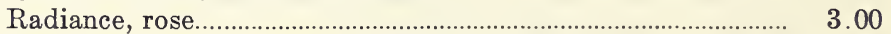

Rose Charm (Waller-Franklin) large bright rose.............................. $\quad 3.00$

Rose Doré (Waller-Franklin) rose pink, suffused orange.................. $\quad 3.00$

Rose Queen, rose pink ................................................................. 2. 2.50

Santa Barbara, dark orange.............................................................. $\quad 3.00$

Showflake, white..................................................................... 3.00

Snowstorm Improved, pure white.............................................. $\quad 2.50$

Snow White, pure white............................................................ $\quad 3.00$

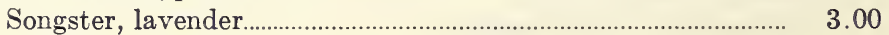




\section{EARLY FLOWERING SPENCER VARIETIES-Continued}

Springsong, salmon pink

Starlight (Waller-Franklin) pale lavender..

Sunray, cerise, cream ground.......................................................... $\quad 7.00$

Superior Pink, rose pink ............................................................. $\quad 3.00$

Sweet Lavender (Waller-Franklin) pure lavender self....................... $\quad 3.00$

The Beauty, fiery rose....................................................................... $\quad 3.00$

Torch, salmon orange........................................................................ 3.00

True Blue, mid blue.................................................................... $\quad 3.00$

Valencia (New) (Waller-Franklin) sunproof orange with robust growth and long stems.

Vulcan, vivid scarlet...................................................................... 3.00

Warbler, rich mauve purple........................................................... $\quad 3.00$

White Harmony, white, black seeded.............................................. $\quad 5.00$

White Rose, pure white............................................................... $\quad 3.50$

Yarrawa, bright rose pink, lighter wings........................................ $\quad 3.00$

Zvolanek's Blue Sensation, clear blue........................................... $\quad 3.00$

Zvolanek's Orange, orange............................................................... 3.00

Zvolanek's Perfection, dark rose.................................................. $\quad 3.00$

Zvolanek's Pink, large pink ........................................................ $\quad 3.00$

Zvolanek's Rose, giant rose pink (select strain)......................... 2.50

Waller-Franklin Formula Mixture "Just a little bit better"...... $\quad 2.00$

Choice Mixed.............................................................................. 1.50

In response to numerous inquiries we submit the following list of twelve Early Flowering Spencer Sweet Peas as being the best in their respective classes, and containing as wide a range of color as possible.

All White, largest pure white.

Amethyst, royal purple.

Aviator, dazzling crimson scarlet.

Blue Bonnet, clear deep blue.

Eldorado, orange.

Fair Maid, blush pink, suffused salmon.

Glitters, cerise.

Grenadier, dazzling scarlet.

Imperial Pink, large clear pink.

Rose Doré, rose pink, suffused orange.

Sweet Lavender, pure lavender self.

Valencia, sunproof orange.

12 BEST

EARLIES. 


\section{NEW AND STANDARD SPENCER VARIETIES}

Adorable, salmon cerise.............................................................. \$ 1.25

Afterglow, reddish mauve, violet wings......................................... 1.00

Ambassador, deep rose pink ............................................................ 1.25

America, red flake, white ground................................................... 1.00

Angele, blue-mauve, suffused pink .................................................. $\quad 1.00$

Annie Ireland, white edged terra cotta pink............................... 1.00

Apple Blossom, rose and pink......................................................... 1.00

Asta Ohn, lavender suffused mauve............................................... 1.00

Austin Frederick Improved, giant lavender................................. 1.00

Avalanche, glistening white of great substance............................ $\quad 1.00$

Barbara, fine salmon.................................................................... 1.00

Blanche Ferry, red and white......................................................... 1.00

Blue Bird, clear blue .................................................................... $\quad 1.00$

Blue Monarch, dark blue............................................................ $\quad 1.00$

Bonfire, cherry pink, cream wings................................................. 1.00

Brilliant Rose, tyrian rose............................................................. 1.00

Campfire, bright scarlet.............................................................. 1.00

Carmelita, rose pink on white ground............................................. $\quad 1.00$

Carnival, rose pink ........................................................................... 1.00

Centaur, deep cerise................................................................... 1.00

Charity, crimson........................................................................ 1.00

Charming, rosy cerise.................................................................... 1.00

Chieftain, satiny mauve................................................................. 1.00

Colne Valley, lavender blue........................................................ $\quad 1.00$

Colorado, bright orange.............................................................. 1.25

Commander Godsall, violet blue.................................................. 1.00

Constance Hinton, mammoth white, black seeded...................... $\quad 1.00$

Coralline, orange cerise.................................................................. 1.00

Countess Spencer, rose pink ....................................................... 1.00

Crimson King, deep crimson.......................................................... $\quad 1.00$

Daffodil, deep cream, black seeded ............................................... $\quad 1.00$

Daisybud Improved, rose pink on white ground......................... $\quad 1.00$

Daventry, deep cerise................................................................. $\quad 1.00$

Defiance (Waller-Franklin) glowing orange scarlet, burnless......... $\quad 1.00$

Delightful, terra cotta cerise............................................................ 1.25

Del Monte, salmon cerise pink ..................................................... 1.00

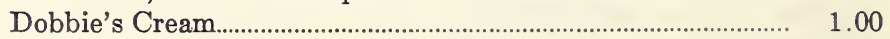

Dora, rosy cerise, cream wings....................................................... $\quad 1.00$

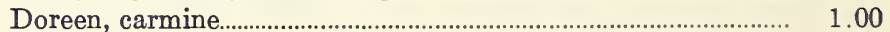

Edith Taylor, rose salmon............................................................. 1.00

Edna May Improved, pure white.................................................... $\quad 1.00$

Elegance, blush lilac suffused pink.............................................. $\quad 1.00$

Elfrida Pearson, large shell pink.................................................. 1.00 


\section{NEW AND STANDARD SPENCER VARIETIES-Continued}

Empire, cream pink.

Etta Dyke, pure white

Faerie Queen, cream, flushed salmon pink.

Fiery Cross, orange cerise.

Flamingo.

Fluffy Ruffles, light cream pink ……………………………..... 1.50

Fortune, rich dark blue..................................................... 1.00

George Shawyer, salmon rose................................................ $\quad 1.00$

Giant Attraction, fawn pink, cream ground............................. $\quad 1.00$

Giant White, black seeded white.............................................. 1.00

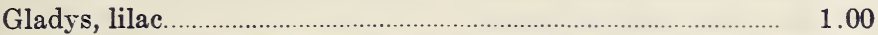

Gleneagles, very large lavender............................................ $\quad 1.00$

Gloriosa, orange scarlet........................................................ 1.00

Glorious, rose cerise............................................................. 1.25

Gold Crest, orange tinted salmon........................................... $\quad 1.00$

Grenadier, geranium red.......................................................... 1.00

Guardsman, pure soft mauve.................................................. 1.00

Guinea Gold, golden orange.................................................... 1.00

Hawlmark Cerise, rose-cerise on cream .................................. 1.00

Hawlmark Lavender, large true lavender..................................... $\quad 1.00$

Hawlmark Pink, deep rose pink .......................................... 1.00

Hawlmark Salmon Pink, salmon pink .................................... 1.00

Hawlmark Scarlet, rich scarlet............................................... 1.00

Heavenly Blue (Waller-Franklin) delphinium blue ............................. 1.00

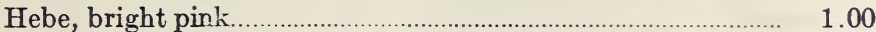

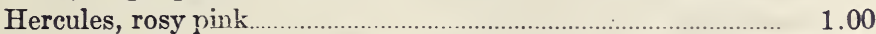

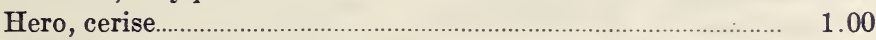

Huntsman, bright scarlet.................................................... 1.00

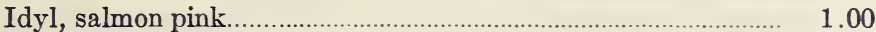

Illuminator, salmon orange, overlaid cerise pink ..................... 1.00

Ivory Picture, ivory cream.................................................. 1.00

Jack Cornwall V. C., dark violet blue..................................... 1.00

Jack Hobbs, flush scarlet pink on cream ground.......................... 1.00

Jean Ireland Improved, cream ground, edged rose .................... 1.00

Joan Ryder, white, black seeded.......................................... 1.00

King Edward, crimson .......................................................... 1.00

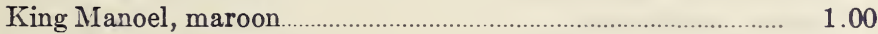

King Mauve, waved mauve .......................................................... 1.00

King White, white

Lavender George Herbert, bluish lavender................................. 1.00

2 L. O., sunproof scarlet...................................................... 1.00

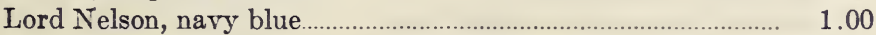

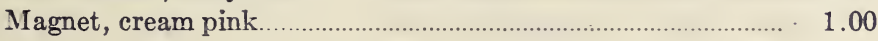




\section{NEW AND STANDARD SPENCER VARIETIES-Continued}

Mammoth, blazing orange scarlet.......................................... \$ $\$ 1.00$

Margaret Atlee, buff pink ............................................................ 1.00

Marjorie Stevenson, deep carmine........................................... 2.00

Mary Pickford, cream pink suffused salmon.............................. $\quad 1.00$

Mascott's Ingman, rosy carmine............................................ 1.00

Mascott's White, solid white...................................................... 1.00

Matchless, cream.......................................................................... 1.00

Mermaid, lavender.................................................................... 1.00

Miss California, salmon cream pink.......................................... $\quad 1.00$

Model, white, black seeded....................................................... 1.00

Montrose, solid rose pink ........................................................... $\quad 2.00$

Mrs. Arnold Hitchcock, pale pink, flushed salmon..................... 1.00

Mrs. A. Searles, rich pink merging into a soft orient red self ... $\quad 1.00$

Mrs. Cuthbertson, rose pink, wings pinkish white.................... 1.00

Mrs. Horace Wright, rosy pink............................................... 1.00

Mrs. Tom Jones, bright delphinium blue ……………………..... $\quad 1.00$

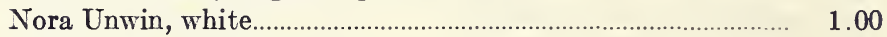

Olympia, deep rich purple.......................................................... 1.00

Peggy, cerise, flushed orange................................................... $\quad 1.00$

Picture, flesh pink suffused creamy apricot............................. $\quad 1.00$

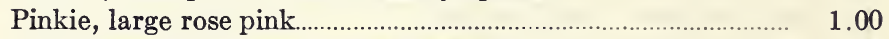

Pink Perfection, rose pink cream ground.................................... $\quad 1.00$

Powerscourt, lavender.......................................................... 1.00

President Harding, peach red.................................................... 1.00

Prince of Orange, deep orange ................................................. 1.25

Ravenswing, dark maroon........................................................... $\quad 1.00$

Red Gauntlet, blood crimson ................................................. 1.00

Renown, carmine rose ........................................................... 1.00

Reflection, clear blue............................................................... 1.25

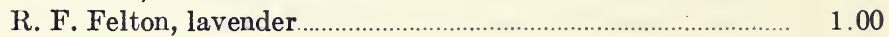

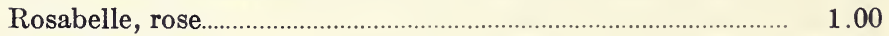

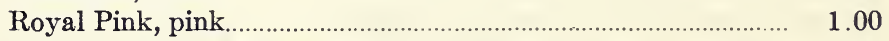

Royal Purple, rich purple....................................................... 1.00

Royal Salute, deep cerise......................................................... $\quad 1.00$

Royal Scot, orange scarlet....................................................... 1.00

Royal Sovereign, orange.............................................................. 1.00

Ruby, carmine ruby............................................................... 1.00

Scarlet Emperor, scarlet......................................................... 1.00

Senator, chocolate flake.............................................................. 1.00

Snowball (Waller-Franklin) dazzling white..................................... $\quad 1.00$

Splendour, red maroon............................................................... $\quad 1.00$

Sunkist, cream picotee edged rose

Sunset, bright rose, amber ground......................................... $\quad 1.00$ 


\section{NEW AND STANDARD SPENCER VARIETIES-Concluded}

Supreme, pale pink.

Sissan, blush pink on cream.

Sybil Henshaw, rich crimson.

Tangerine Improved, glowing orange.

The Cardinal (Waller-Franklin) poppy scarlet.

The Lady Eveline, rosy mauve.

The President, orange scarlet.

The Prince, crimson.

The Sultan, black velvety maroon.

Tom Webster, medium deep blue.

Valentine, blush pink.

Vectis, best white.

Venus, cream pink suffused salmon.

Vermilion Brilliant, scarlet.

Warrior, maroon.

Wedgwood, azure blue.

Wembley, lavender suffused plumbago blue.

What Joy, cream

White Spencer.

Winnie Morse, lilac flushed rose.

Wizard, salmon scarlet.

Youth, large white, pink picotee.

SILVER GILT MEDAL MIXTURE (Waller-Franklin). This mixture is made from a formula that has been perfected after years of experimenting and is a wonderful blend of only the best and brightest Spencers. This mixture contains all the worthwhile novelties of recent years 


\section{SWEET PEAS}

\section{COLOR CLASSIFICATION}

In view of the numerous recent Sweet Pea introductions, it seems necessary to set up a classification according to color.

Every year we make careful color comparisons of the older varieties and the latest introductions and are in an eminent position to judge their respective merits.

We now take pleasure in submitting a color classification of the Standard Spencers.

The variety we consider best in each color class is indicated by an asterisk $(*)$.

BICOLOR (Light)

Blanche Ferry

*Bonfire

BICOLOR (Dark)

*Angele

Captain of the Blues

BLUE

${ }^{*}$ Blue Bird

Heavenly Blue

Mrs. Tom Jones

Tom Webster

BLUE (Light)

Colne Valley

*Gleneagles

Mermaid

BLUE (Dark)

Commander Godsall

*Fortune

Jack Cornwall V. C.

BLUSH (Pink)

*Valentine

BLUSH (Lilac)

*Elegance
CARMINE

*Brilliant Rose

Doreen

Mascott's Ingman

Renown

\section{CERISE}

Centaur

Daventry

Hero

*Royal Salute

CERISE (Rose)

*Charming

Coralline

Illuminator

Peggy

CERISE (Salmon)

Adorable

Hawlmark Cerise

${ }^{*}$ Mrs. A. Searles

CERISE (Scarlet)

Campfire

Defiance

Flamingo

*Grenadier

2 L. O.

Royal Scot 
COLOR CLASSIFICATION-Continued

The variety we consider best in each color class is indicated by an asterisk $\left({ }^{*}\right)$.

CREAM

Daffodil

Matchless

*What Joy

CREAM PINI (Light)

Comrade

Mary Pickford

Mrs. Arnold Hitchcock

*Susan

Venus

CREAM PINK (Deep)

Giant Attraction

Idyl

*Magnet

Picture

CRIMSON

Charity

${ }^{*}$ Crimson King

Sybil Henshaw

\section{FLUSHED}

Carmelita

Faerie Queen

*Jack Hobbs

Mrs. Horace Wright

LAVENDER (Rosy)

Austin Frederick Imp.

${ }^{*}$ R. F. Felton

LAVENDER (Light)

Hawlmark Lavender

*Tembley

\section{LAVENDER (Lilac)}

Gladys

*Powerscourt

MARBLED, FLAKED

AND STRIPED

America

Senator

MAROON (Dark)

Ravenswing

*The Sultan

Warrior

MAROON (Red)

*Splendour

MAUVE

${ }^{*}$ Chief tain

Guardsman

King Mauve

The Lady Eveline

ORANGE

*Royal Sovereign

Tangerine Improved

ORANGE (Deep)

*Colorado

Guinea Gold

Prince of Orange

Wizard

ORANGE (Pink)

George Shawyer

*Royal Pink

ORANGE (Scarlet)

Gloriosa

*Mammoth

The President 


\section{COLOR CLASSIFICATION-Concluded}

The variety we consider best in each color class is indicated by an asterisk (*).

PICOTEE (Cream Ground)

Ivory Picture

Jean Ireland Imp.

*Sunkist

PICOTEE (White Ground)

Annie Ireland

*Youth

PINK (Pale)

Daisybud Improved

Elfrida Pearson

*Supreme

PINK (Rose, Deep)

Hebe

Hercules

*Pinkie

PINK (Salmon, Deep)

*Del Monte

Hawlmark Pink

Hawlmark Salmon Pink

Miss California

PURPLE

*Olympia

Royal Purple
ROSE

Rosabelle

*Sunset

\section{SALMON}

Barbara

*Gold Crest

\section{SCARLET}

Hawlmark Scarlet

*Huntsman

2 L. $O$.

The Cardinal

\section{WHITE}

*Avalanche

Edna May Improved

King White

Mascott's White

Snowball

Vectis

WHITE (Tinted)

Constance Hinton

Joan Ryder

*Model 


\section{CUPID SPENCER VARIETIES}

Little Nell (Waller-Franklin) pink and white.

Midshipmite (Waller-Franklin) white edged violet blue............. $\quad 2.00$

Peter Pan (New) (Waller-Franklin) deep bright pink ................... 2.00

Rosebud (Waller-Franklin) apricot pink ...................................... $\quad 2.00$

Mixed, above varieties.............................................................. $1.7 j$

\section{GRANDIFLORA VARIETIES}

America, white ground, red flake................................................ 45

Black Knight, maroon ...........................................................

Blanche Ferry, red and white..................................................

Dorothy Eckford Improved, white ......................................... . .45

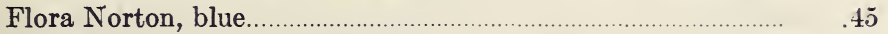

Hon. Mrs. E. Kenyon, primrose $\ldots \ldots \ldots \ldots \ldots \ldots \ldots \ldots \ldots \ldots \ldots \ldots \ldots \ldots \ldots \ldots \ldots \ldots \ldots \ldots \ldots \ldots \ldots \ldots \ldots \ldots \ldots . . . . .45$

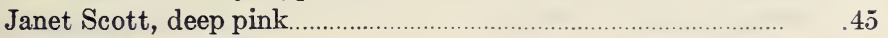

King Edward VII, red.......................................................... . 45

Lady Grisel Hamilton, lavender

Lord Nelson, navy blue................................................................ $\quad .45$

Miss Willmott, orange pink

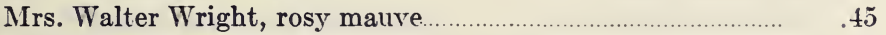

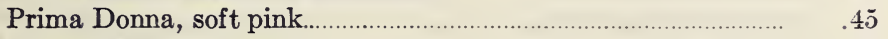

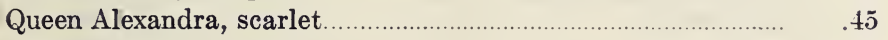

Eckford's Choice Mixed................................................................

\section{CUPID GRANDIFLORA VARIETIES}

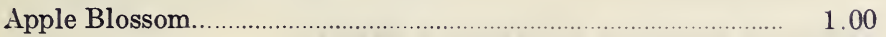

Blanche Ferry .................................................................. 1.00

Captain of the Blues ......................................................... 1.00

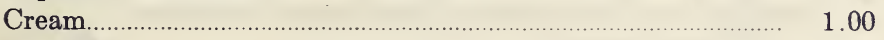

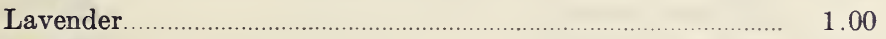

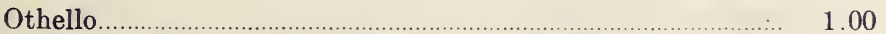

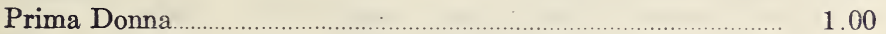

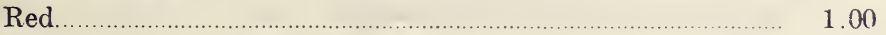

White

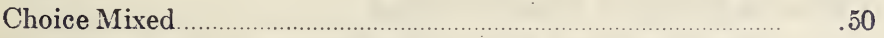


SWEET WILLIAM, Annual Single Mixed $\$ 1.50$

$\$ 5.00$

THUNBERGIA, Mixed, fine climber.

TRITOMA (Red-hot Poker)

Hybrida Mirabilis, blooms first year.

$3.00 \quad 10.00$

Early Hybrids Mixed.

TROPAEOLUM Canariense (Canary Bird Vine).

\section{VERBENA HYBRIDA}

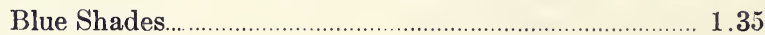

Dark Blue

Defiance, bright scarlet.

Firefly, scarlet, white eye.

Italian Striped.

Lucifer (Waller-Franklin) vivid scarlet.

Lutea Improved, deep creamy yellow.

Mayflower, pink

Pink and Rose Shades.

Purple Mantle, purple.

Purple Shades.

White..

Choice Mixed.

\section{VERBENA HYBRIDA COMPACTA ERECTA}

Chamois, pale chamois tinted buff.

Fireball (New) (Waller-Franklin) dwarf compact six inches high, bright scarlet.

Compacta Dwarf Mixed.

Royal Bouquet Mixed, upright large flowers in brilliant mixture suitable for pot culture..

\section{VERBENA HYBRIDA GRANDIFLORA}

A magnificent new strain of Verbenas, of robust compact habit and free blooming. The trusses are immense, the individual florets measuring an inch in diameter.

Blue (Waller-Franklin) white eye............................................. $3.00 \quad 10.00$

Coccinea (Waller-Franklin) scarlet, small eye $\ldots \ldots \ldots \ldots \ldots \ldots \ldots \ldots . . . .3 .00 \quad 3 . . .100$

Colossea Rose Shades......................................................... $3.00 \quad 10.00$

Etna (New) (Waller-Franklin) Paul Crampbell geranium red with creamy yellow eye................................... $3.00 \quad 10.00$ Giant Salmon Pink............................................................... $3.00 \quad 10.00$ 


\section{GROWERS OF “MERIT” FLOWER SEEDS}

\section{VERBENIA HYBRIDA GRANDIFLORA-Continued}

Lucifer (Waller-Franklin) vivid scarlet. $\$ 3.00 \$ 10.00$

Luminosa (Waller-Franklin) luminous flame pink shading to salmon 3.00 10.00

Rosea Stellata (Waller-Franklin) rose pink, white eye....... 3.00 10.00

Rose Cardinal (Waller-Franklin) with large white eye........ 3.00 10.00

Royale (New) (Waller-Franklin) royal blue, creamy yellow eye

10.00

Violacea Stellata, deep violet, white eye. 3.00 10.00

White (Waller-Franklin). $3.00 \quad 10.00$

Grandiflora Mixed (Gigantea).

$2.50 \quad 8.00$

VERBENA VENOSA, purplish heliotrope.

1.25

\section{VIRGINIAN STOCK}

Compact Rose

White

Mixed

\section{WALLFLOWER ANNUAL DOUBLE}

Early Wonder Mixed (New) a fine cut flower, suitable for pot culture.

\section{WALLFLOWER ANNUAL SINGLE}

Blood Red............................................................................ . .30

Creamy White ........................................................................ . .30

Paris Brown

Yellow

Mixed.

XERANTHEMUM, Double Mixed

\section{ZINNIA GIANT DOUBLE DAHLIA FLOWERED}

Canary Bird, primrose yellow.

Crimson Monarch, deep crimson.

Dream, lavender turning to purple

Exquisite, light rose with deep rose center.

Old Rose, old rose shade.

Oriole, orange and gold.

Polar Bear, white.

Scarlet Flame, bright scarlet.

Waller-Franklin Formula Mixture, "Just a little bit better" 


\section{ZINNIA GIANT DOUBLE MAMMOTH}

Cerise Queen, cerise rose

$\$ 2.50 \quad \$ 8.00$

Enchantress, light rose with deep rose center................. 2.50 $\quad 8.00$

Lemon Queen, lemon orange.............................................. 2.50 8.00

Miss Wilmott, soft pink …………………………….......... 2.50 8.00

Orange King, burnt orange..................................................... 2.50 2.00

Orange Queen, golden yellow................................................. 2.50 $\quad 8.00$

Purity, white..................................................................... $2.50 \quad \$ .00$

Rose Queen, bright rose................................................... $2.50 \quad 8.00$

Scarlet Gem, glowing scarlet............................................... $2.50 \quad$ \$.00

Choice Mixed........................................................................ $2.10 \quad 7.00$

\section{ZINNIA GIANT DOUBLE (GRAINDIFLORA ROBUSTA)}

Canary Yellow...................................................................... $1.80 \quad 6.00$

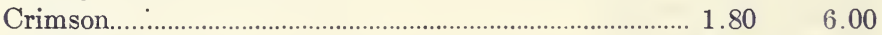

Deep Flesh.............................................................................. $1.80 \quad 6.00$

Golden Yellow................................................................... $1.80 \quad 6.00$

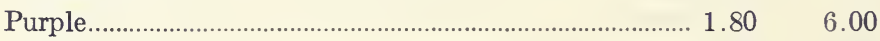

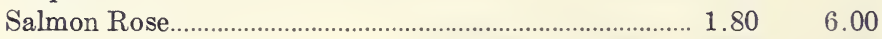

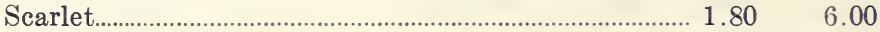

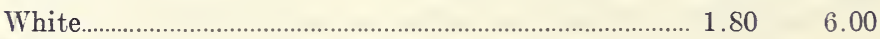

Waller-Franklin Formula Mixture, "Just a little bit

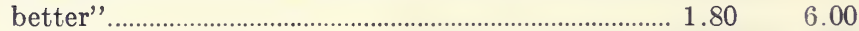

Choice Mixed, all colors........................................................ $1.25 \quad 4.00$

ZINNIA GIANT DOUBLE PICOTEE MIXED ..................... $2.50 \quad 8.00$

ZINNIA GIANT DOUBLE QUILLED MIXED (Achievement).

ZINNIA ELEGANS DOUBLE CUT AND COME AGAIN-A group with double, perfectly formed, medium sized flowers bred with long stems; exceptionally fine for cut flowers. This is a "Cut and Come Again" group and is especially fine for massing in the garden and produces a continuous succession of cut flowers throughout the Summer.

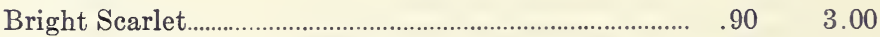

Canary Yellow......................................................................... $\quad .90 \quad 3.00$

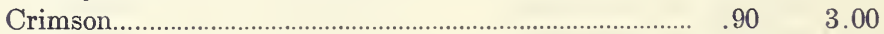

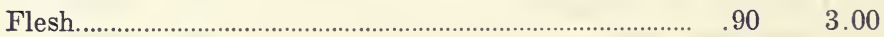

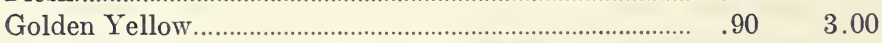

Salmon Rose........................................................................ $1.25 \quad 4.00$

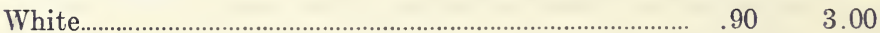

Waller-Franklin Formula Mixture, "Just a little bit better"... 
GROWERS OF "MERIT" FLOWER SEEDS

FLOWER SEEDS

Price Price

$1 / 4 \mathrm{lb}$. per lb.

ZINNIA ELEGANS DOUBLE LILLIPUT

Canary Yellow

Golden Orange.

Salmon Rose

Scarlet

White

1.50

5.00

Mired.

ZINNIA DOUBLE GRACILLIMA

Red Riding Hood (Coccinea).

ZINNIA DOUBLE HAAGEANA FYBRIDA, Mixed, charm-

ing hybrids in a great variety of color.

\section{WALLER-FRANKLIN WILD GARDEN MIXTURE}

A well balanced mixture of hardy annuals, suitable for planting in odd corners and racant lots. Excellent for gift packets.

\section{WALLER-FRANKLIN CUT FLOWER GARDEN MIXTURE}

A mixture of hardy annuals blended to give a continuous succession of cut flowers from early summer until late fall 


\section{IN CONCLUSION}

As the foregoing list shows, we specialize exclusively in Annual Flower Seeds including a few perennials.

We are, in fact, specialists in growing Annual Flower Seeds and the heart of our business is composed of fifty acres of Experimental, Stock Seed Production and Test Grounds operated by a staff of experts. Here are developed pure line strains of varieties and special formulae for mixtures. In all, we farm over two thousand acres of land, and every operation, from the time the seed is planted until it is harvested, threshed, cleaned and shipped to our customers, is supervised by experts.

We solicit your continued patronage on the merit of our seeds and the fairness of our prices.

WALLER-FRANKLIN SEED COMPANY 
MEMORANDA 
MEMORANDA 
MEMORANDA 


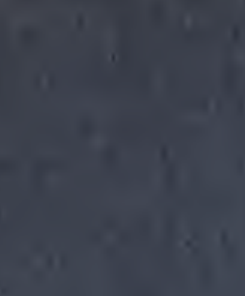

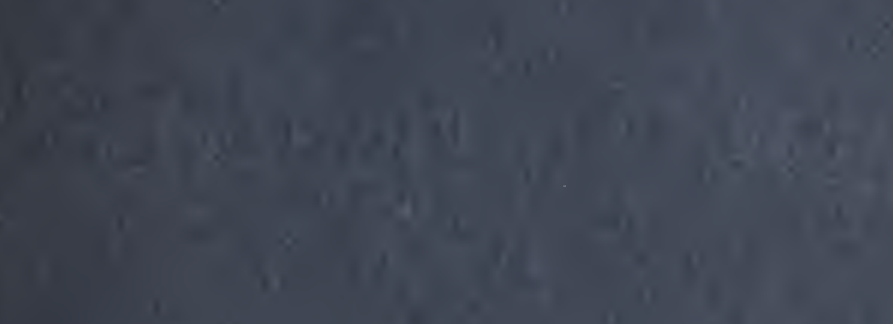

$+1$

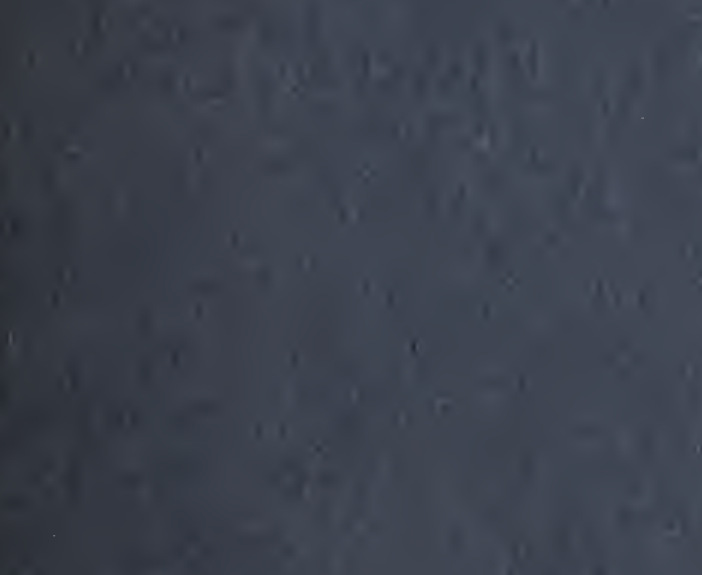

(1)

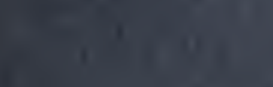

w

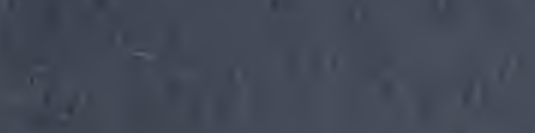

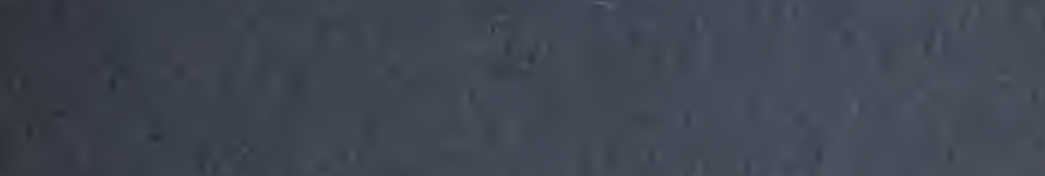

6

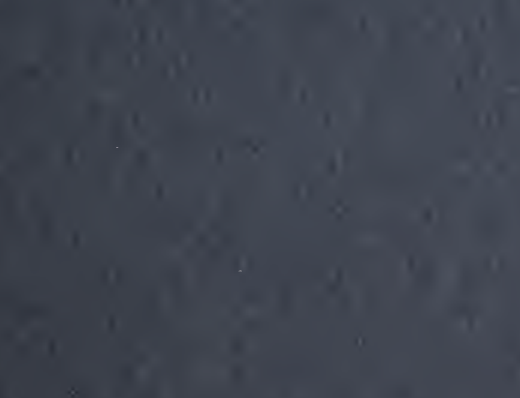

(a)

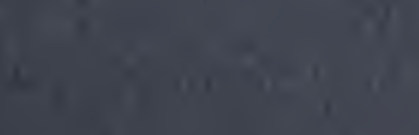

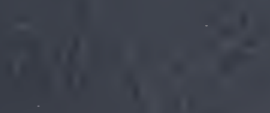

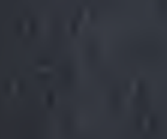$$
\text { +1 - +1 } 1
$$ 


\section{WALLER-ERANKIITN}

1980 